\title{
Extension of multi-commodity closed-loop supply chain network design by aggregate production planning
}

\author{
Leena Steinke $^{1} \cdot$ Kathrin Fischer ${ }^{1}$
}

Received: 30 June 2015/Accepted: 17 October 2016/Published online: 14 November 2016

(c) The Author(s) 2016. This article is published with open access at Springerlink.com

\begin{abstract}
In this work the influence of production and capacity planning on decisions regarding facility location, distribution quantities and component remanufacturing (and vice versa) in a closed-loop supply chain network (CLSCN) with multiple make-to-order products is studied. A mathematical model, the facility location, capacity and aggregate production planning with remanufacturing (FLCAPPR) model, for designing the CLSCN, for planning capacities at the facilities and for structuring the production and distribution system of the network cost-optimally, is formulated. It consists of a facility location model with component remanufacturing over multiple time periods, which is extended by capacity and production planning on an aggregate level. The problem is solved for an example set of data which is based on previous CLSC research in the copier industry. In a numerical study the effect of the extended planning approach on the decision to process returned products is determined. Furthermore, the FLCAPPR model is solved for different returned product quantities and numbers of periods in the planning horizon to study the influence on the network design and on the procuring, production and distribution quantities. It turns out that decisions regarding the locations of and the
\end{abstract}

This article is part of a focus collection on "Robust Manufacturing Control: Robustness and Resilience in Global Manufacturing Networks".

Leena Steinke

1.steinke@tuhh.de

Kathrin Fischer

kathrin.fischer@tuhh.de

1 Institute of Operations Research and Information Systems, Hamburg University of Technology, Schwarzenbergstr. 95, 21073 Hamburg, Germany capacity equipment at facilities and decisions regarding the production and distribution system are interdependent; therefore, they have to be managed jointly. Furthermore, it is shown that the decision to process returned products and use remanufactured components in the production does depend not only on the costs, but also on the quantity of returned products and the length of the planning horizon.

Keywords Closed-loop supply chain management . Network design · Remanufacturing · Reverse logistics . Aggregate production planning · Capacity planning

\section{Introduction}

Supply chains with product recovery differ, depending on the characteristics of the product, the recovery activity which is used and whether this activity is done by the original equipment manufacturer or a third party [6]. In general, supply chains with product recovery can be distinguished into open-loop and closed-loop supply chains (CLSC). If there is hardly any connection of the forward and return product flows, the supply chain is open-loop and the forward and reverse product flows are managed separately. The forward product flow can be described by the traditional supply chain management theory, and the reverse product flow is planned independently by reverse supply chain management [25]. If the forward and return product flows are related, e.g. customers supply their used products as production inputs, the supply chain is closedloop. In this case, often an integrated management of both flows is necessary to achieve an optimized CLSC; for further details see $[8,9]$.

In this work, a supply chain is studied which is closed by component remanufacturing. Remanufacturing is also 
called value-added recovery, since it describes a series of operations which restore the value of a product after usage [11]. A supply chain with remanufacturing is extended by the following activities: collecting, cleaning and testing returned products. Then, remanufacturable products are disassembled into components, which are remanufactured, e.g. repaired or refurbished. After testing these components, they are reassembled and sold in secondary markets as remanufactured items or reintegrated to the original supply chain and used as as-new items [11], as in the supply chain studied in this work. High-value products, e.g. copiers and automobiles, are suitable for component remanufacturing. A further discussion of product characteristics that enable remanufacturing can be found in [18].

Whether the supply chain is open- or closed-loop, product recovery forces supply chain management (SCM) to consider a reverse product flow. In addition to the planning, realization and control of all operations, production, inventory and distribution quantities and information flows from the product origin to the point of consumption, all problems concerning the way back through the supply chain, i.e. after consumption, have to be considered in a $\mathrm{SCM}$ with product recovery. These decision problems can be differentiated regarding their planning horizon: some are made on a yearly basis and determine the framework for decisions, which are made on a weekly or monthly basis [26]. Then again, these decisions constrain the operational decisions, which occur every day [20].

The network design, decisions regarding the product and material programme, supplier selection, collection strategy, take-back arrangements and supply chain coordination are strategic decision problems and belong to long-term planning. Decision problems regarding inventory management and production planning are tactical and have a mid-term planning horizon. Operative decision problems, as disassembly planning, material requirement plans, scheduling and routing in the remanufacturing shop have a short-term planning horizon [4, 5, 7, 28].

In order to achieve an optimized CLSC the tactical planning has to be considered by strategic management $[14,21,26]$. Long-range forecasts of aggregate product demand are the input for strategic planning [5]. They are used by the mathematical model developed in this work to derive a cost-optimally network design, i.e. cost-optimal facility locations and capacity equipment, with cost-optimal procuring, transportation, production and storage quantities. The quantities are planned on an aggregate level; therefore, fluctuations of data are neglected and the modelling approach is deterministic.

In the facility location problem (FLP), facilities are located and quantities of goods are allocated and distributed in the network a cost-optimal way, e.g. in [2]. In the special case of a CLSN with reverse product flows these models support the procuring decision, i.e. when to recover returned products and use them as production inputs, as well, e.g. in [8, 9].

In the literature so far, location/allocation models in a CLSCN consider opening costs of product recovery facilities, but costs for volume capacity and costs for installing technology or hiring workforce for the operations at the respective facilities of the network, especially for product recovery operations, are neglected. However, to determine a cost-optimal procurement policy, i.e. to decide when to recover returned products and use the resulting items as production inputs instead of new items procured from suppliers, these costs have to be included.

Since remanufacturing is a labour-intensive operation (see [13] for an extended discussion), labour hour costs are relevant for the decision to process returned products. The well-established aggregate production planning (APP)framework is used to plan the production and workforce at facilities cost-optimally in this work. In APP, the length of the planning horizon is usually between 6 and 24 months [3] and quantities are planned on an aggregate level. In the following this APP-approach is described and a multi-period facility location problem extended by capacity and aggregate production planning is developed.

The consideration of different product compositions, component remanufacturing and component commonality is possible with this modelling approach, and the influence of different return rates can be investigated. Hence, different realistic SC settings can be captured.

The rest of the paper is organized as follows: relevant selected literature regarding network design and aggregate production planning is presented and discussed in the next section. Here, the differences between other contributions and the approach taken in this work are also discussed. The CLSCN and the production planning problem are presented in detail in Sect. 3. Afterwards the planning problem is described mathematically in Sect. 4. In Sect. 5, it is solved for an example data set and the results are presented. Furthermore, a sensitivity analysis is performed and selected results are discussed in Sect. 5, too. Finally, conclusions and possibilities for further research are stated.

\section{Literature review}

Networks with product recovery are mathematically optimized by extending the classical Warehouse Location Problem (WLP) to capture reverse product flows. Mostly these problems are described by Mixed Integer Programming (MIP) and Mixed Integer Linear Programming (MILP) models. In the following, selected papers are discussed, which present the state of research, and have influenced this study. A more detailed review of network 
design literature concerning supply chains with product recovery is offered in [1].

As one of the first, Marin and Pelegrin [19] study a network with reverse product flows: customers get products at plants and return them to plants. The objective is to find the optimal plant location and shipping quantities, such that the costs for opening facilities and for transportation are minimized. Marin and Pelegrin's [19] model is an uncapacitated Facility Location Problem (FLP), whereas the other models discussed in the following are capacitated FLP.

Following [19], in this work it is assumed that customers return their products to those facilities from which the products are distributed. Unlike [19], in the network studied here, these facilities are not plants, but facilities for distribution and collection of products, called distribution and collection centres (DCCs). Furthermore, in [19] a single product type is considered, whereas in this work multiple product types are studied.

A remanufacturing network with multiple product types is examined by Jayaraman et al. [15]. Used products are shipped from collection zones to remanufacturing centres, where they can be remanufactured or stored. Remanufactured products are distributed, i.e. are used to fulfil customer demand, or stored. The shipping quantities between collection zones, customers and remanufacturing/distribution locations are to be determined optimally; the objective is cost minimization.

In this work, following [15], it is assumed that returned products can be stored at remanufacturing centres. As in [15], the storage capacity at remanufacturing facilities is assumed to be limited. Additional to storage capacities, capacities for operations at facilities are planned in this work, too.

Operative capacity equipment is studied by Schultmann et al. [24]. The model by [24] allocates the optimal operative capacity equipment to open facilities of an existing reverse supply chain. The capacity at facilities is needed for operations, as e.g. inspection and sorting, of multiple product types. The objective is to minimize costs, caused by capacity equipment, production and distribution quantities.

Unlike in [24], in this work a closed-loop system is studied, i.e. in addition to reverse product flows, forward product flows are considered. Fleischmann et al. [8] and Fleischmann et al. [9] study such a closed-loop system as a three echelon network, consisting of warehouses, plants and test centres, where products are recovered. These facilities have to be located optimally, and the quantities of the forward and reverse product flows of the network are to be determined such that costs for opening, transport and for unsatisfied demand and not-collected returned products are minimized under capacity limitations for the product flows between facilities of different network echelons.
Salema et al. [23] extend the model from [9] to study multiple product types. Furthermore, in [23] the product flow capacity at facilities is limited by maximum and minimum capacity bounds for the facilities.

As in $[9,23]$, the CLSCN studied in this work has three facility levels. Here, the three facility levels of the CLSCN are DCCs, plants and suppliers and remanufacturing centres, both delivering components to plants. The production system of the CLSCN consists of two stages: at the first stage components are delivered from remanufacturing centres or from suppliers to plants, where they are assembled to products. This way, component remanufacturing, unlike product remanufacturing as in $[9,15,19,23]$, with different product and component types and component commonality in the assembly of different product types can be modelled.

In contrast to $[9,15,23]$, the capacity for storage and product and component flows at facilities are decision variables, i.e. have to be determined out of a parameter range and induce costs. In addition, capacity for the operations at facilities is determined by the model stated in this work. Hence, the influence of different capacity types on the facility location decisions and on the decision to produce, remanufacture, store or distribute is studied.

All studies discussed above have a single time period planning horizon. Following $[15,21]$ consider inventory in a single time period planning facility location problem and study the trade-off between storing and distributing products. Further discussions of inventory in distribution networks and the interdependence of inventory, transportation and facility location can be found in [21] and different approaches to extend facility location problems by inventory management can be found in [26].

In this work, the planning horizon of the CLSCN design problem is modified to a multi-period setting, as in [22] and [30]. Pishvaee and Torabi [22] use a multi-objective approach to combine cost minimization of the CLSCN with the minimization of delivery tardiness for the single product case. In this work, the objective is to minimize costs and the production and capacity planning problem at open facilities in a network with multiple commodities is considered in a MILP-approach.

In this work, a FLP for a closed-loop supply chain with component remanufacturing is extended by production planning on an aggregate level, using the idea of APP, as in Steinke and Fischer [30]. In APPs, the different products are aggregated to product types and the capacities are not product specific, but are summarized and stated in common units, e.g. labour hours. APP is used to determine costoptimal manufacturing and storage plans, which match the limited means in terms of workforce or working stations, respectively, and production input with forecasted demand [20]. The planning horizon of an APP can vary; usually it 
consists of 6-24 months, [3]. In particular, when adjustments of capacities are allowed in each period, the periods have to be sufficiently long.

Jayaraman [16] studies the production planning problem of a company, which offers recovered mobile phones for a secondary market. He states the Remanufacturing APP (RAPP) model, which minimizes costs by determining the optimal disassembly, disposal, remanufacturing, procurement and storage quantities under fixed workforce capacities.

In this work, the approach of [16] is followed to model the production system. While in [16] the reverse product flow is managed, here, a closed-loop system is studied, and the model is extended accordingly, i.e. the remanufactured components are reintegrated into the original supply chain instead of being shipped to secondary markets. Moreover, in contrast to [16], capacities in volume units and labour hours at facilities are not fixed but can be adjusted over the planning horizon.

In the RAPP proposed by [16] only one site for remanufacturing is considered, whereas in this work, multiple possible facility locations exist. Hence, the APP for a closed-loop system is integrated into a FLP. Extending a yearly FLP for a CLSCN with component recovery by an APP on a monthly basis leads to a model with extensive solution times. Furthermore, the considered capacities cannot be adjusted within one month; especially, decisions regarding the volume capacity are made on a strategic level. Therefore, also the APP is extended and the APP is modelled for a strategic, yearly, planning horizon.

With such an extended strategic planning model, decisions regarding the location of facilities and their capacity equipment for operations and storage can be studied jointly. Furthermore, different product and component types are considered and the interdependence of processing, storing and distributing them is examined. Moreover, by considering capacity costs and operative capacity in addition to storage capacity, the cost effects of the decisions regarding the returned products, i.e. if they are remanufactured, stored or disposed, are captured completely unlike in facility location problems without capacity and production planning.

Following [9, 19, 23, 30], a fixed relation between demand and returned products is assumed in this work; the returned product quantity is determined as a fraction of the sold product quantity. As in [30], the CLSCN is studied over multiple time periods, and hence, there is a time lag between the selling and the returning of a product. In [30] it is assumed that products are returned by customers after one period of usage. However, products can stay longer with the customers, i.e. the residence time of a product, defined as the number of periods a product is used by a customer, can be longer. Furthermore, products are returned not only in a specific period following the buying, but in all subsequent periods of the planning horizon. In this work, the model in [30] is extended to capture these aspects.

Moreover, the FLCAPPR model developed in this work determines cost-optimal volume capacities and optimal workforce size at the facilities for every period. In contrast, in [31] the capacity planning is integrated in a more simplified way, such that overcapacities can occur: whenever a facility is opened, its volume capacity and workforce are set to their respective upper limits and adjusted to the actual required levels only in the last period. Furthermore, while in [30] total costs are minimized, here discounting is considered in the objective function, too.

\section{Problem description}

In this section the network structure of the CLSCN with component remanufacturing is introduced and the respective planning problem is described in detail.

The CLSCN consists of nodes, which represent customers and facilities with their operations, and arrows, which show the flows of multiple commodities through the network, see Fig. 1. There are five different types of nodes: costumers, DCCs, remanufacturing centres, plants and suppliers. Customers demand different product quantities in each period, and they return their products to DCCs in a later period, i.e. it is assumed that a known fraction of products shipped to customers in one period is returned in a later period of the planning horizon. The residence time of products can be different, but there is a given number of periods the product has to stay with a customer before it is returned and considered as remanufacturable. The minimum residence time can be interpreted as the minimum number of periods a product is in full working condition. Demand quantities are assumed as deterministic and known; therefore, the returned product quantities are deterministic and known, too. Demand is lost whenever it is not met, i.e. it cannot be backlogged.

The CLSCN consists of three facility levels: DCCs, plants, remanufacturing centres and suppliers. The latter are summarized to one level since both provide components. Supplier locations are given, whereas the locations of DCCs, remanufacturing centres and plants have to be determined. These facilities can be opened in one period and then remain open or are closed in a later period. It is assumed that once a facility is closed, it cannot be opened again.

Capacities at facilities are determined in volume and labour hours. The volume capacity restricts the volume of commodity flows passing a facility and, if existent, the volume of stocked products and components, respectively. 


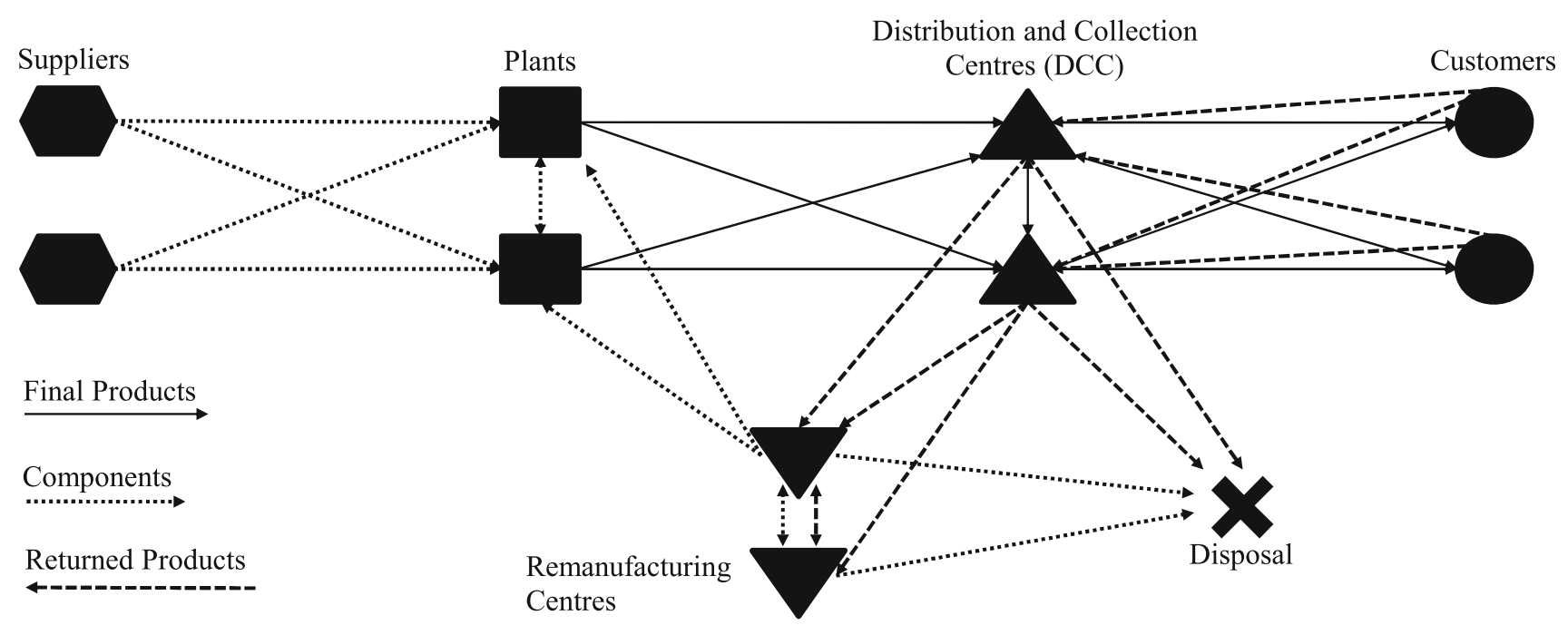

Fig. 1 Closed-loop supply chain network

The labour hour capacity limits the available hours of the workforce needed for remanufacturing and assembly at remanufacturing centres and plants, and for handling products at DCCs.

Capacity levels at facilities are determined once a facility is opened and can be adjusted in a later period, i.e. they can be expanded or reduced in fixed steps in every period.

The product and component flows through the network are described by three different types of arrows, see Fig. 1 . The solid arrows show the forward product flows, which are shipped from plants to DCCs and further to the customer locations. The dotted arrows describe the component flows leaving suppliers or remanufacturing centres, respectively, to plants. The dashed arrows represent the reverse product flows, i.e. the flows from customers to DCCs and from DCCs to remanufacturing centres. In this CLSCN redistribution is possible, i.e. products and components can be shipped between facilities of the same type.

DCCs are bi-directional facilities, because products flow through DCCs to customers and customers return used products to DCCs. At DCCs returned products are collected, visually inspected and, afterwards, they are shipped either to remanufacturing centres or to the disposal unit.

The decomposition of returned products into components and the remanufacturing of those components to an as-new condition is performed at remanufacturing centres. It is assumed that components can be remanufactured repeatedly in the planning horizon, i.e. the limited number of possible remanufacturing cycles for components is not reached. However, there is a known and constant fraction of components that cannot be remanufactured to the quality standards of as-new components with a reasonable given effort and therefore has to be disposed. Moreover, at remanufacturing centres it is possible to store returned products, instead of remanufacturing them immediately.

At plants, components are assembled to products of different types. Product types differ regarding their combination of components, i.e. at least one component in the product composition has to be different in different products. Components can be product type-specifically or commonly used among different product types. They are shipped from suppliers or remanufacturing centres to plants and can be held on inventory at plants. No final products are stored in the studied network and products are assembled only if an order exists (MTO). Since the planning horizon is strategic, no lead-times for operations or transport are considered.

For each planning period the demand and return product quantities are known, while facility locations and capacity equipment at the facilities, as well as procurement, transportation, production and inventory quantities, have to be determined with the objective of total cost minimization. To support these decisions, the planning problem is formulated as a MILP, presented in the next section.

\section{The facility location, capacity and aggregate production planning with remanufacturing problem}

In this section, the planning problem described above, the Facility Location, Capacity and Aggregate Production Planning with Remanufacturing (FLCAPPR) Problem, is stated and explained using the notation listed in Tables 1,2 and 3, presented below. The model presented here is an extension of the model given in [30], as described in Sect. 2 above. 
Table 1 Definition of relevant sets

\begin{tabular}{ll}
\hline Set & Definition \\
\hline$C$ & Set of components, $c \in C$ \\
$F$ & Set of potential plants, $f \in F$ \\
$F D$ & $F \cup\{D\}$, set of potential plants and the disposal unit $D$ \\
$K$ & Set of customer locations, $k \in K$ \\
$P$ & Set of products, $p \in P$ \\
$R$ & Set of potential remanufacturing centres, $r \in R$ \\
$R D$ & $R \cup\{D\}$, set of potential remanufacturing centres and the disposal unit $D$ \\
$T$ & Set of time periods, $t \in T$ \\
$V$ & Set of potential DCCs, $v \in V$ \\
$Z$ & Set of suppliers for components, $z \in Z$ \\
\hline
\end{tabular}

Table 2 Definition of relevant variables

\begin{tabular}{|c|c|}
\hline Variable & Definition \\
\hline Capt & Number of capacity steps at open facility $y$ in period $t$ (in $\mathrm{m}^{3}$ ), $\forall y \in F \cup V \cup R, t \in T$ \\
\hline CCapt & Expansion or reduction of capacity steps at facility $y$ in period $t$ (in $\mathrm{m}^{3}$ ), $\forall y \in F \cup V \cup R, t \in T$ \\
\hline$C C a p D_{t}^{y}$ & Reduction of capacity steps at facility $y$ in period $t$ (in $\left.\mathrm{m}^{3}\right), \forall y \in F \cup V \cup R, t \in T$ \\
\hline$C C a p U_{t}^{y}$ & Expansion of capacity steps at facility $y$ in period $t$ (in $\left.\mathrm{m}^{3}\right), \forall y \in F \cup V \cup R, t \in T$ \\
\hline$E I_{c}^{f}$ & Quantity of $c$ remaining in the inventory of plant $f$ at the end of the last planning period, $\forall f \in F, c \in C$ \\
\hline$E I_{x}^{r}$ & $\begin{array}{l}\text { Quantity of } x \text { remaining in the inventory of remanufacturing centre } r \text { at the end of the last planning period, } \\
\forall r \in R, x \in C \cup P\end{array}$ \\
\hline$E X I_{x t}^{y w}$ & $\begin{array}{l}\text { Quantity of } x \text { transported from facility } y \text { to facility } w \text { of the same echelon in period } t \\
\forall y, w \in F \cup V \cup R: y \neq w, x \in C \cup P, t \in T\end{array}$ \\
\hline$H_{t}^{y}$ & $\left\{\begin{array}{c}1, \quad \text { if facility } y \text { is closed in period } t \\
\forall y \in F \cup V \cup R, t \in T \\
0, \quad \text { otherwise }\end{array}\right.$ \\
\hline$I_{c t}^{f}$ & Quantity of $c$ remaining at plant $f$ at the end of period $t, \forall f \in F, c \in C, t \in T$ \\
\hline$I_{x t}^{r}$ & Quantity of $x$ remaining at remanufacturing centre $r$ at the end of period $t, \forall r \in R, x \in C \cup P, t \in T$ \\
\hline$L C a p_{t}^{y}$ & Workforce available at facility $y$ in period $t, \forall y \in F \cup V \cup R, t \in T$ \\
\hline$U_{p t}^{k}$ & Number of unmet demand for product $p$ of customer $k$ in period $t, \forall k \in K, p \in P, t \in T$ \\
\hline$X_{x t}^{y}$ & Quantity of $x$ processed in facility $y$ in period $t, \forall y \in F \cup R, x \in C \cup P, t \in T$ \\
\hline$X_{x t}^{y w}$ & Quantity of $x$ transported from facility $y$ to facility $w$ in period $t, \forall y, w \in F D, V, R D, Z: y \neq w, x \in C \cup P, t \in T$ \\
\hline$Y_{E t}^{y}$ & $\left\{\begin{aligned} 1, \quad \text { if facility } y & \text { is opened in period } t \\
\forall y & \in F \cup V \cup R, t \in T \\
0, & \text { otherwise }\end{aligned}\right.$ \\
\hline$Y_{t}^{y}$ & $\left\{\begin{array}{c}1, \quad \text { if facility } y \text { is open in period } t \\
\forall y \in F \cup V \cup R, t \in T \\
0, \quad \text { otherwise }\end{array}\right.$ \\
\hline$Y C C a p U_{t}^{y}$ & $\left\{\begin{array}{c}1, \quad \text { if capacity of facility } y \text { is increased in } \\
\text { period } t, \forall y \in F \cup V \cup R, t \in T \\
0, \\
\text { otherwise }\end{array}\right.$ \\
\hline
\end{tabular}

The objective function of the FLCAPPR problem minimizes the discounted total costs of the CLSCN over multiple time periods. As the model combines multi-period facility location, capacity and aggregate production planning, the objective function consists of cost terms for opening, running and closing facilities, for the volume capacity equipment and the labour force at open facilities, for processing and storing goods at facilities, for procuring components at suppliers, for transporting goods in the network and for disposing returned products and components. 
Table 3 Definition of relevant parameters

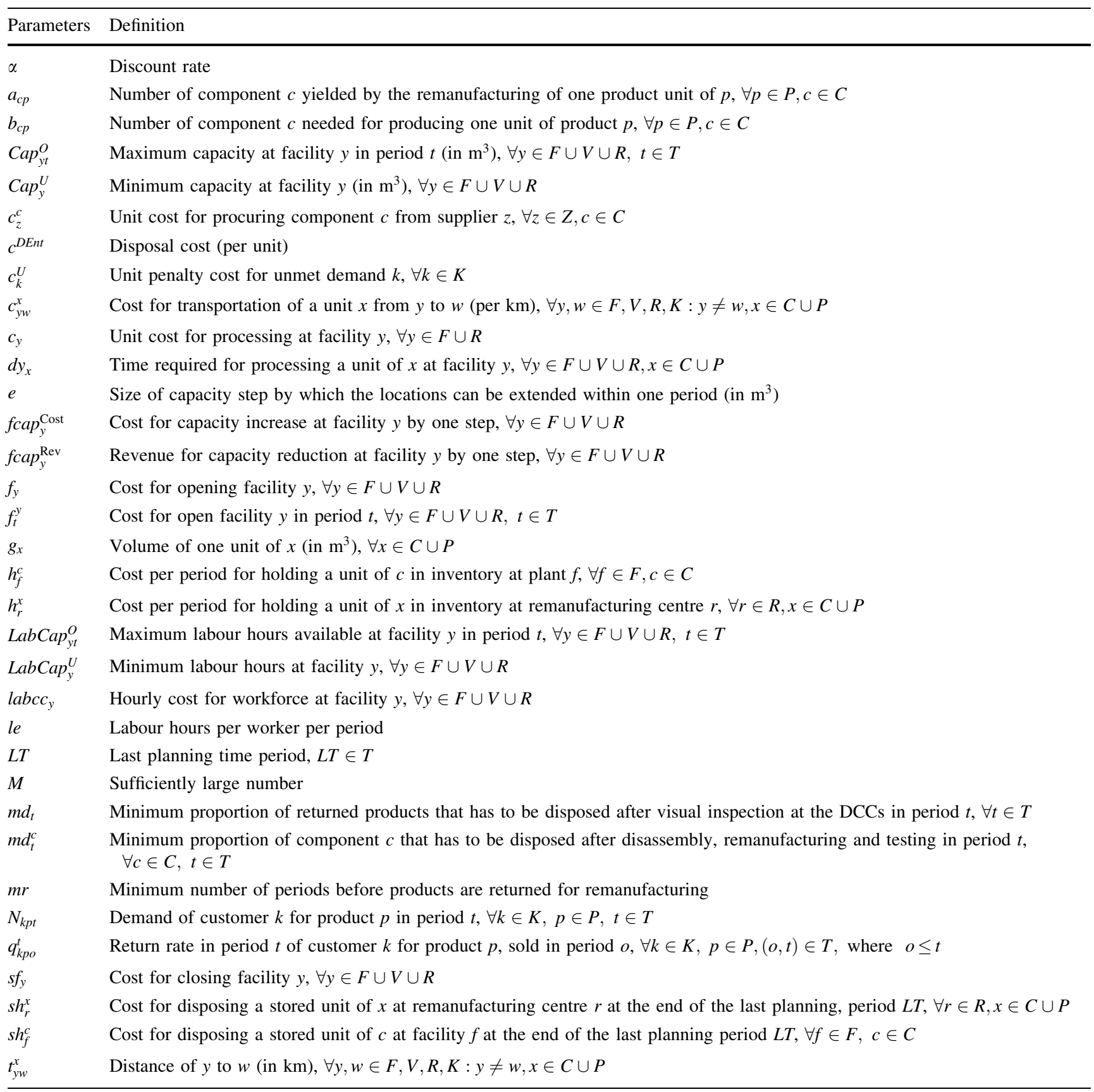


The discounted total costs are described by the following objective function (1). For the sake of clarity the objective function is split up into three different cost functions. The first cost function presents the costs induced by multi-period facility location, the second function includes the costs resulting from capacity planning, and the costs of aggregate production planning are described by the third function. Below the functions are introduced followed by the respective explanations.

$$
\min \mathrm{OF}=\mathrm{OF}_{1}+\mathrm{OF}_{2}+\mathrm{OF}_{3}
$$

with

$$
\begin{aligned}
& \mathrm{OF}_{1}=\sum_{t \in T}\left(( 1 / ( 1 + \alpha ) ^ { t } ) \cdot \left(\sum_{r \in R} f_{r} \cdot Y_{E t}^{r}\right.\right. \\
& +\sum_{v \in V} f_{v} \cdot Y_{E t}^{v}+\sum_{f \in F} f_{f} \cdot Y_{E t}^{f} \\
& +\sum_{r \in R} f_{t}^{r} \cdot Y_{t}^{r}+\sum_{v \in V} f_{t}^{v} \cdot Y_{t}^{v}+\sum_{f \in F} f_{t}^{f} \cdot Y_{t}^{f} \\
& +\sum_{r \in R} s f_{r} \cdot H_{t}^{r}+\sum_{v \in V} s f_{v} \cdot H_{t}^{v}+\sum_{f \in F} s f_{f} \cdot H_{t}^{f} \\
& +\sum_{z \in Z, c \in C, f \in F}\left(c_{z f}^{c} \cdot t_{z f}^{c}+c_{z}^{c}\right) \cdot X_{c t}^{z f} \\
& +\sum_{r \in R, c \in C, f \in F} c_{r f}^{c} \cdot t_{r f}^{c} \cdot X_{c t}^{r f} \\
& +\sum_{f \in F, v \in V, p \in P} c_{f v}^{p} \cdot t_{f v}^{p} \cdot X_{p t}^{f v} \\
& +\sum_{v \in V,} c_{k \in K, p \in P} c_{v k}^{p} \cdot t_{v k}^{p} \cdot X_{p t}^{v k}+\sum_{k \in K, p \in P} c_{k}^{U} \cdot U_{p t}^{k} \\
& +\sum_{v \in V, k \in K, p \in P} c_{k v}^{p} \cdot t_{v k}^{p} \cdot X_{p t}^{k v} \\
& +\sum_{v \in V, r \in R, p \in P} c_{v r}^{p} \cdot t_{v r}^{p} \cdot X_{p t}^{v r} \\
& +c^{D E n t} \cdot\left(\sum_{v \in V, p \in P} X_{p t}^{v D}+\sum_{r \in R, c \in C} X_{c t}^{r D}\right) \\
& +\sum_{c \in C,(r, s) \in R: r \neq s} c_{r s}^{c} \cdot t_{r s}^{c} \cdot E X I_{c t}^{r s} \\
& +\sum_{p \in P,(r, s) \in R: r \neq s} c_{r s}^{p} \cdot t_{r s}^{p} \cdot E X I_{p t}^{r s} \\
& +\sum_{c \in C,(f, i) \in F: f \neq i} c_{f i}^{c} \cdot t_{f i}^{c} \cdot E X I_{c t}^{f i} \\
& \left.+\sum_{p \in P,(v, j) \in V: v \neq j} c_{v j}^{p} \cdot t_{v j}^{p} \cdot E X I_{p t}^{v j}\right)
\end{aligned}
$$

In the multi-period FLP studied in this work, the facilities can be opened in one period and in the later periods they can stay open or are closed. The variables $Y_{E t}^{y}, Y_{t}^{y}$ and $H_{t}^{y}$ describe the respective state of a facility. The costs for opening, i.e. building, a facility, occur just once and are listed in line one and two. For every period in which a facility remains open it induces costs; these costs are captured by the terms in line three. In line four the costs for closing a facility are stated.

The cost terms in the next line are for procuring and shipping components from suppliers to plants. Costs for transporting components from remanufacturing centres to plants are listed in line six.

The transportation costs of the forward product flow, the flow of products from plants to DCCs and from DCCs to customers, and the penalty costs for unsatisfied demand are stated in line seven and eight.

The cost terms in line nine and ten are the shipping costs of the reverse product flow, i.e. the flow of products which are returned by customers to DCCs and flow further in the network to remanufacturing centres or to the disposal unit. In the latter case, costs for disposing occur. The disposal costs for returned products and remanufactured components are listed in line eleven. The costs for distributing products or components, respectively, on the same facility level are listed in line $12-15$.

$$
\begin{aligned}
\mathrm{OF}_{2}= & \sum_{t \in T}\left(( 1 / ( 1 + \alpha ) ^ { t } ) \cdot \left(\sum_{r \in R} f c a p_{r}^{\mathrm{Cost}} \cdot C \operatorname{Cap} U_{t}^{r}\right.\right. \\
& +\sum_{f \in F} f c a p_{f}^{\mathrm{Cost}} \cdot C \operatorname{Cap} U_{t}^{f}+\sum_{v \in V} f_{c a p}^{\mathrm{Cost}} \cdot C \operatorname{Cap} U_{t}^{v} \\
& +\sum_{r \in R} f c a p_{r}^{\mathrm{Rev}} \cdot C C a p D_{t}^{r}+\sum_{f \in F} f c a p_{f}^{\mathrm{Rev}} \cdot C C a p D_{t}^{f} \\
& \left.+\sum_{v \in V} f c a p_{v}^{\mathrm{Rev}} \cdot C C a p D_{t}^{v}\right)
\end{aligned}
$$

At facilities, certain volume capacities in $\mathrm{m}^{3}$ are available and they can be increased or decreased within one period. These adjustments induce costs or revenues, as reflected by the cost terms in line 1 and 2 or revenues in line 3 and 4, respectively.

$$
\begin{aligned}
\mathrm{OF}_{3}= & \sum_{t \in T}\left(( 1 / ( 1 + \alpha ) ^ { t } ) \cdot \left(\sum_{r \in R, c \in C} c_{r} \cdot X_{c t}^{r}\right.\right. \\
& +\sum_{f \in F, p \in P} c_{f} \cdot X_{p t}^{f}+l e \cdot\left(\sum_{r \in R} l a b c c_{r}^{\text {Cost }} \cdot L C a p_{t}^{r}\right. \\
& \left.+\sum_{f \in F} l a b c c_{f}^{\text {Cost }} \cdot L C a p_{t}^{f}+\sum_{v \in V} l a b c c_{v}^{\text {Cost }} \cdot L C a p_{t}^{v}\right) \\
& +\sum_{r \in R, p \in P} h_{r}^{p} \cdot I_{p t}^{r}+\sum_{r \in R, c \in C} h_{r}^{c} \cdot I_{c t}^{r} \\
& \left.+\sum_{f \in F, c \in C} h_{f}^{c} \cdot I_{c t}^{f}\right) \\
& +\left(1 /(1+\alpha)^{L T}\right) \cdot\left(\sum_{f \in F, c \in C} s h_{f}^{c} \cdot E I_{c}^{f}\right. \\
& \left.+\sum_{r \in R, p \in P} s h_{r}^{p} \cdot E I_{p}^{r}+\sum_{r \in R, c \in C} s h_{r}^{c} \cdot E I_{c}^{r}\right)
\end{aligned}
$$


Remanufacturing components at remanufacturing centres and assembling products at plants induces costs, see lines one and two. Labour hours of the workforce are needed for performing the respective operations at the facilities. The respective costs occur in every period and are stated in line 2 and 3.

Holding products and components at remanufacturing centres and holding components at plants induces costs, which are captured by the cost terms in lines 4 and 5 .

The costs stated in lines 1-5 occur in every period, and hence, these costs have to summed up over the planning horizon. At the end of the planning horizon the remaining items on stock at the facilities are disposed, and the respective cost terms are stated in the last two lines.

In the following, the constraints of the problem are presented, but before that, important variables are explained.

The variables $Y_{E t}^{y}, Y_{t}^{y}$ and $H_{t}^{y}$ are interrelated. If a facility is opened in one period, then it is running in this period; therefore, both variables $Y_{E t}^{y}$ and $Y_{t}^{y}$ take value 1 and $H_{t}^{y}$ is zero.

In the next period this facility can be still open, then $Y_{t+1}^{y}=1$ and $Y_{E t+1}^{y}=0$, because the facility is already opened, and $H_{t+1}^{y}=0$. However, the open facility can be closed in $t+1$, then $H_{t+1}^{y}$ takes value 1 , and $Y_{t+1}^{y}=Y_{E t+1}^{y}=0$.

It is assumed that a facility cannot be opened again after it is closed. The constraints (2)-(6) define these interrelations.

$\sum_{t \in T} Y_{E t}^{y} \leq 1 \quad \forall y \in F \cup R \cup V$

A facility can be opened just once in the planning horizon.

$Y_{E 1}^{y}=Y_{1}^{y} \quad \forall y \in F \cup R \cup V$

If a facility is opened in the first planing period, it is open in period 1 .

$$
\begin{aligned}
& \sum_{t \in T: t \leq s}\left(Y_{E t}^{y}-H_{t}^{y}\right)=Y_{s}^{y} \\
& \quad \forall y \in F \cup R \cup V, s \in T: s>1
\end{aligned}
$$

If a facility is opened and not closed in one of the periods $t \leq s$, where $(s, t) \in T$, then the facility is open in period $s$.

$$
Y_{t-1}^{y}-Y_{t}^{y} \leq H_{t}^{y} \quad \forall y \in F \cup R \cup V, t \in T: t>1
$$

These constraints indicate the closing of facilities by comparing the opening indicator variables of two successive periods.

$$
\sum_{t \in T} H_{t}^{y} \leq 1 \quad \forall y \in F \cup R \cup V
$$

Closing of facilities is allowed to happen once within the planning horizon.

The next set of constraints, constraints (7)-(19), describes the forward and reverse product flows in the network and the inventory balance at plants and remanufacturing centres.

$\sum_{v \in V} X_{p t}^{v k}+U_{p t}^{k}=N_{k p t} \quad \forall k \in K, p \in P, t \in T$

Products are shipped from DCCs to satisfy demand of customer $k$ for product $p$ in period $t$. Unsatisfied demand is captured by $U_{p t}^{k}$.

$$
\begin{aligned}
& \sum_{v \in V} X_{p t}^{k v}=0 \quad \forall t \in\{1, \ldots, m r-1\}, k \in K, p \in P \\
& \sum_{v \in V} X_{p t}^{k v}=\sum_{o=1}^{t}\left(q_{k p o}^{t} \cdot \sum_{v \in V} X_{p o}^{v k}\right) \\
& \forall t \in\{m r, \ldots, T\}, k \in K, p \in P
\end{aligned}
$$

After $m r$ periods, products can be returned for the first time. In period $t$ a proportion of products sold in period $o$, $q_{k p o}^{t}$, is returned to DCCs. Every returned product is collected in DCCs.

$$
\begin{aligned}
I_{c t}^{f}= & I_{c t-1}^{f}+\sum_{z \in Z} X_{c t}^{f f}+\sum_{r \in R} X_{c t}^{r f} \\
& +\sum_{i \in F: i \neq f} E X I_{c t}^{i f}-\sum_{i \in F: i \neq f} E X I_{c t}^{f i}-X_{c t}^{f} \\
& \forall f \in F, c \in C, t \in T: t>1
\end{aligned}
$$

These constraints represent the inventory balance equations for components at plants.

$$
\begin{aligned}
I_{p t}^{r}= & I_{p t-1}^{r}+\sum_{v \in V} X_{p t}^{v r}+\sum_{s \in R: s \neq r} E X I_{p t}^{s r} \\
& -\sum_{s \in R: s \neq r} E X I_{p t}^{r s}-X_{p t}^{r} \\
& \forall r \in R, p \in P, t \in T: t>1
\end{aligned}
$$

The inventory balance equations for returned products at remanufacturing centres are stated in (11).

$$
\begin{aligned}
I_{c t}^{r}= & I_{c t-1}^{r}+X_{c t}^{r}+\sum_{s \in R: s \neq r} E X I_{c t}^{s r} \\
& -\sum_{s \in R: s \neq r} E X I_{c t}^{r s}-\sum_{f \in F} X_{c t}^{r f} \\
& \forall r \in R, c \in C, t \in T: t>1
\end{aligned}
$$

Components can be stocked at remanufacturing centres, too. The inventory balance is determined by the equations (12). 


$$
\begin{aligned}
I_{c 1}^{f}= & \sum_{z \in Z} X_{c 1}^{z f}+\sum_{r \in R} X_{c 1}^{r f}+\sum_{i \in F: i \neq f} E X I_{c 1}^{i f} \\
& -\sum_{i \in F: i \neq f} E X I_{c 1}^{f i}-X_{c 1}^{f} \quad \forall f \in F, c \in C \\
I_{x 1}^{r}= & X_{x 1}^{r}+\sum_{s \in R: s \neq r} E X I_{x 1}^{s r}-\sum_{s \in R: s \neq r} E X I_{x 1}^{r s} \\
& -\sum_{f \in F} X_{x 1}^{r f} \quad \forall r \in R, x \in C \cup P
\end{aligned}
$$

The constraints (13) and (14) define the balance of the respective inventory at the end of the first period.

$$
\begin{array}{ll}
I_{c L T}^{f}=E I_{c}^{f} & \forall f \in F, c \in C \\
I_{x L T}^{r}=E I_{x}^{r} & \forall r \in R, x \in C \cup P
\end{array}
$$

Products and components remaining in the respective inventories at the end of the last planning period, $L T$, are captured by (15) and (16).

$$
\begin{gathered}
\sum_{f \in F} X_{p t}^{f v}+\sum_{j \in V: j \neq v} E X I_{p t}^{j v}-\sum_{j \in V: j \neq v} E X I_{p t}^{v j} \\
=\sum_{k \in K} X_{p t}^{v k} \quad \forall v \in V, t \in T, p \in P
\end{gathered}
$$

Since no inventory at DCCs is allowed, every product entering a DCC in period $t$ also has to leave it in period $t$.

$$
\sum_{v \in V} X_{p t}^{f v}=X_{p t}^{f} \quad \forall f \in F, t \in T, p \in P
$$

There is no product inventory at plants, i.e. every assembled product in a plant in period $t$ is shipped to DCCs in the same period.

$\sum_{r \in R D} X_{p t}^{v r}=\sum_{k \in K} X_{p t}^{k v} \quad \forall v \in V, t \in T, p \in P$

Every returned product is shipped from DCCs either to remanufacturing centres or to the disposal unit $\mathrm{D}$.

The constraints (20) and (21) define the disposal quantities in the network.

$X_{p t}^{v D} \geq m d_{t} \cdot \sum_{k \in K} X_{p t}^{k v} \quad \forall v \in V, t \in T, p \in P$

At least a proportion of $m d_{t}$ of the returned products has to be disposed in period $t$, because of failing the inspection at the DCCs.

$$
m d_{t}^{c} \cdot X_{c t}^{r} \leq X_{c t}^{r D} \quad \forall r \in R, t \in T, c \in C
$$

After remanufacturing, at least a proportion of $m d_{t}^{c}$ of the components does not comply with the requirements for asnew components and is disposed.
The following constraint sets, the constraints (22) and (23), describe the disassembly and assembly operations at the remanufacturing centres and plants, respectively.

$$
X_{c t}^{r}=\sum_{p \in P} a_{c p} \cdot X_{p t}^{r} \quad \forall r \in R, t \in T, c \in C
$$

The number of as-new components, derived by disassembling returned products and remanufacturing the respective components, is defined by the equations above.

$$
X_{c t}^{f}=\sum_{p \in P} b_{c p} \cdot X_{p t}^{f} \quad \forall f \in F, t \in T, c \in C
$$

The number of components required for product assembly at plants is defined by these equations.

At facilities capacity in labour hours and volume are considered and have to be planned over the planning horizon. The next sets of constraints, the constraints (24)(43), describe the capacity planning.

$$
\begin{aligned}
\sum_{p \in P} d V_{p} \cdot\left(\sum_{k \in K}\left(X_{p t}^{k v}+X_{p t}^{v k}\right)\right) \leq l e \cdot L C a p_{t}^{v} \\
\forall v \in V, t \in T
\end{aligned}
$$

The capacity level in terms of labour hours at facilities is the product of one worker's labour hours per period, le, multiplied by the workforce available in $t$, determined by $L C a p_{t}^{v}$ for DCCs. The constraints above adhere that the labour hours needed for handling products at DCCs do not exceed the available capacity level.

$\sum_{c \in C} d R_{c} \cdot X_{c t}^{r} \leq l e \cdot L C a p_{t}^{r} \quad \forall r \in R, t \in T$

The labour hours used for remanufacturing at a remanufacturing centre cannot exceed the respective available capacity.

$\sum_{p \in P} d F_{p} \cdot X_{p t}^{f} \leq l e \cdot L C a p_{t}^{f} \quad \forall f \in F, t \in T$

At plants, capacity in terms of labour hours is needed for assembling products. It is limited by the capacity level at a plant.

$$
\begin{gathered}
\operatorname{LabCap}_{y}^{U} \cdot Y_{t}^{y} \leq l e \cdot L C a p_{t}^{y} \leq L a b C a p_{y t}^{O} \cdot Y_{t}^{y} \\
\forall y \in F \cup V \cup R, t \in T
\end{gathered}
$$

The capacity in labour hours at an open facility is restricted by upper and lower bounds, forced by operations and the availability of workers.

$$
\sum_{c \in C} g_{c} \cdot I_{c t}^{f} \leq e \cdot \operatorname{Cap}_{t}^{f} \quad \forall f \in F, t \in T
$$


The volume capacity at the facilities is a multiple of $e$. The volume of components stocked at an open plant cannot exceed its available volume capacity.

$$
\sum_{c \in C} g_{c} \cdot I_{c t}^{r}+\sum_{p \in P} g_{p} \cdot I_{p t}^{r} \leq e \cdot \operatorname{Cap}_{t}^{r} \quad \forall r \in R, t \in T
$$

At an open remanufacturing centre, the volume of stored products and components has to comply with the volume capacity.

$$
\begin{aligned}
& \sum_{c \in C} g_{c} \cdot\left(\sum_{z \in Z} X_{c t}^{z f}+\sum_{r \in R} X_{c t}^{r f}+\sum_{i \in F: i \neq f} E X I_{c t}^{i f}\right) \\
& \leq e \cdot \operatorname{Cap}_{t}^{f} \quad \forall f \in F, t \in T
\end{aligned}
$$

The volume of components flowing into a plant is restricted by the available volume capacity.

$\sum_{p \in P} g_{p} \cdot X_{p t}^{f v} \leq e \cdot \operatorname{Cap}_{t}^{f} \quad \forall f \in F, t \in T$

The product flow through a plant adheres to the volume capacity restriction of a plant.

$$
\begin{gathered}
\sum_{c \in C} g_{c} \cdot\left(X_{c t}^{r}+\sum_{s \in R: s \neq r} E X I_{c t}^{s r}\right)+\sum_{p \in P} g_{p} . \\
\left(\sum_{v \in V} X_{p t}^{v r}+\sum_{s \in R: s \neq r} E X I_{p t}^{s r}\right) \leq e \cdot C a p_{t}^{r}
\end{gathered}
$$

$$
\forall r \in R, t \in T
$$

The volume of the components and products flowing into a remanufacturing centre is limited by its volume capacity restriction.

$$
\begin{gathered}
\sum_{c \in C} g_{c} \cdot\left(X_{c t}^{r f}+\sum_{s \in R: s \neq r} E X I_{c t}^{r s}\right)+\sum_{p \in P} g_{p} . \\
\left(\sum_{s \in R: s \neq r} E X I_{p t}^{r s}\right) \leq e \cdot \operatorname{Cap}_{t}^{r} \\
\forall r \in R, t \in T
\end{gathered}
$$

The volume of the components and products leaving a remanufacturing centre has to be less or equal than the respective capacity level.

$$
\begin{gathered}
\sum_{p \in P} g_{p} \cdot\left(\sum_{f \in F} X_{p t}^{f v}+\sum_{k \in K} X_{p t}^{k v}+\sum_{j \in V: j \neq v} E X I_{p t}^{j v}\right) \\
\leq e \cdot C a p_{t}^{v} \quad \forall v \in V, t \in T
\end{gathered}
$$

The volume of products flowing through a DCC has to comply with its capacity.

$$
\begin{array}{r}
\operatorname{Cap}_{y}^{U} \cdot Y_{t}^{y} \leq e \cdot \operatorname{Cap}_{t}^{y} \leq \operatorname{Cap}_{y t}^{O} \cdot Y_{t}^{y} \\
\forall y \in F \cup V \cup R, t \in T
\end{array}
$$

The volume capacity level of a facility is a multiple of $e$ and is limited by given upper and lower bound.

$$
\begin{aligned}
& C a p_{t}^{y}-C_{a p}^{y}=C C a p_{t}^{y} \\
& \forall y \in F \cup V \cup R, t \in T: t>1
\end{aligned}
$$

Volume capacity at facilities can be expanded or reduced within one period.

$$
C a p_{1}^{y}=C_{C a p}^{y} \quad \forall y \in F \cup V \cup R
$$

In the first planning period, the number of capacity steps at a facility is identical to the capacity expansion carried out in period 1 .

$$
\begin{gathered}
\operatorname{CCap}_{t}^{y} \leq \operatorname{Cap}_{y t}^{O} \cdot \operatorname{YCCap}_{t}^{y} \\
\forall y \in F \cup V \cup R, t \in T
\end{gathered}
$$

The variable $Y C C a p U_{t}^{y}$ takes value 1 , if the respective variable $C C a p_{t}^{y}$ is bigger than zero, i.e. the capacity of facility $y$ is increased in period $t$.

$$
\begin{gathered}
C C a p_{t}^{y}-C \operatorname{Cap} U_{t}^{y} \leq \operatorname{Cap}_{y t}^{O} \cdot\left(1-Y C \operatorname{Cap} U_{t}^{y}\right) \\
\forall y \in F \cup V \cup R, t \in T
\end{gathered}
$$

Capacity increase is assigned to the variable $C \operatorname{Cap} U_{t}^{y}$.

$$
\begin{gathered}
C C_{a p} U_{t}^{y} \leq \operatorname{Cap}_{y t}^{o} \cdot Y \text { CCap }_{t}^{y} \\
\forall y \in F \cup V \cup R, t \in T
\end{gathered}
$$

The upper capacity bound of a facility limits the capacity increase.

$$
\begin{gathered}
C C a p_{t}^{y}-C_{C a p D}^{y} \leq \operatorname{Cap}_{y t}^{O} \cdot Y C \operatorname{Cap} U_{t}^{y} \\
\forall y \in F \cup V \cup R, t \in T
\end{gathered}
$$

The variable $C \operatorname{Cap} D_{t}^{y}$ captures the capacity decrease.

$$
\begin{gathered}
\operatorname{CCapD}_{t}^{y} \geq-\operatorname{Cap}_{y t}^{O} \cdot\left(1-\operatorname{YCCap}_{t}^{y}\right) \\
\forall y \in F \cup V \cup R, t \in T
\end{gathered}
$$

Capacity decrease at a facility cannot be higher than the respective upper capacity bound.

$$
Y_{C C a p} U_{t}^{y} \leq Y_{t}^{y} \quad \forall y \in F \cup V \cup R, t \in T
$$

Capacity can just be increased at an open facility. 
$U_{p t}^{k}, X_{p t}^{k v}, X_{p t}^{v k}, X_{p t}^{v r}, X_{c t}^{z f}, X_{c t}^{r}, X_{p t}^{r}, X_{c t}^{v f}, X_{p t}^{f v}$,

$X_{p t}^{f}, X_{c t}^{f}, E X I_{c t}^{r s}, E X I_{p t}^{r s}, E X I_{c t}^{f i}, E X I_{p t}^{v j}, I_{c t}^{f}, I_{c t}^{r}$,

$I_{p t}^{r}, E I_{c}^{f}, E I_{c}^{r}, E I_{p}^{r}, \operatorname{Cap}_{t}^{r}, \operatorname{Cap}_{t}^{f}, \operatorname{Cap}_{t}^{v}, \operatorname{CCap}_{t}^{y}$,

$L \operatorname{Cap}_{t}^{r}, L \operatorname{Cap}_{t}^{f}, L \operatorname{Cap}_{t}^{v} \in \mathbb{Z}^{+} \forall p \in P, c \in C$,

$r \in R D, k \in K, v \in V, f \in F D, z \in Z, t \in T$

Unsatisfied demand, the flows of products or components between different echelons and between facilities of one echelon, the produced units, the units on stock, the capacities at the facilities and the capacity increase are described by positive integer variables.

$C \operatorname{Cap} t_{t}^{y} \in \mathbb{Z} \quad \forall y \in F \cup V \cup R, t \in T$

Variables describing the change of capacities at facilities are integers and can be positive or negative.

$C \operatorname{CapD} D_{t}^{y} \in \mathbb{Z}^{-} \quad \forall y \in F \cup V \cup R, t \in T$

The variables that determine the volume capacity decrease are negative integer variables.

$$
\begin{gathered}
Y^{y}, Y_{t}^{y}, H_{t}^{y}, Y C \operatorname{Cap}_{t}^{y} \in\{0,1\} \\
\forall y \in F \cup V \cup R, t \in T
\end{gathered}
$$

Binary variables indicate the opening and closing of facilities and the capacity increase.

\section{Numerical analysis}

In this section, the previously stated FLCAPPR model is solved for an example data set. At first, the example, its data and the solution are described, and then, the sensitivity of the network to changes in the data is studied. The influence of the cost parameters on the decision to remanufacture is explored. Furthermore, the robustness of the solution with respect to the quantity of returned products and the length of the planning horizon is examined by varying the return rate and the number of periods in the planning horizon. Selected interesting results are discussed.

\subsection{Initial setting and solution}

In the copier industry, CLSCNs as the one described in Sect. 3 can be found. At copier manufacturer Xerox, for example, "remanufactured parts are put onto the assembly line for reuse in brand new copiers" [17]. Fleischmann et al [8] study the facility location problem in a CLSCN of a European copier remanufacturer for a single-period planning horizon.

In this paper a CLSCN for the copier industry in Germany is to be designed. The product demand is assumed to be bundled in the fifteen biggest German cities. As in [8] the total product demand is assumed as 10 units per 1000 inhabitants, where the number of inhabitants is taken from [29]. Furthermore, it is assumed that demand occurs in every period of the planning horizon, since, as in [8], one planning period equates to one year.

Costumers demand two different product types, $P 1$ and $P 2$. Demand for $P 1$ and $P 2$ is assumed as equally high, i.e. 500 units of $P 1$ and $P 2$ are required, in every period.

It is possible to open DCCs and remanufacturing centres at the demand locations. Suppliers and possible plant locations are to be found only in the five biggest German cities. In Fig. 2 the customer locations with their demand in product units per planning period are given. Furthermore, the suppliers and all possible locations for DCCs, remanufacturing centres and plants are shown.

In the initial example used in this work, it is assumed that demand for $P 1$ - and $P 2$-products is known for five years and remains constant over the planning horizon. Later on, a sensitivity analysis is presented which includes a study on the impact of varying the length of the planning horizon.

The assembly of each product type requires one specific component, $M 1$ for $P 1$ - and $M 3$ for $P 2$-products, and one component, $M 2$, which is commonly used for both product types. For simplicity the disassembly process is assumed to be the reverse of the assembly process.

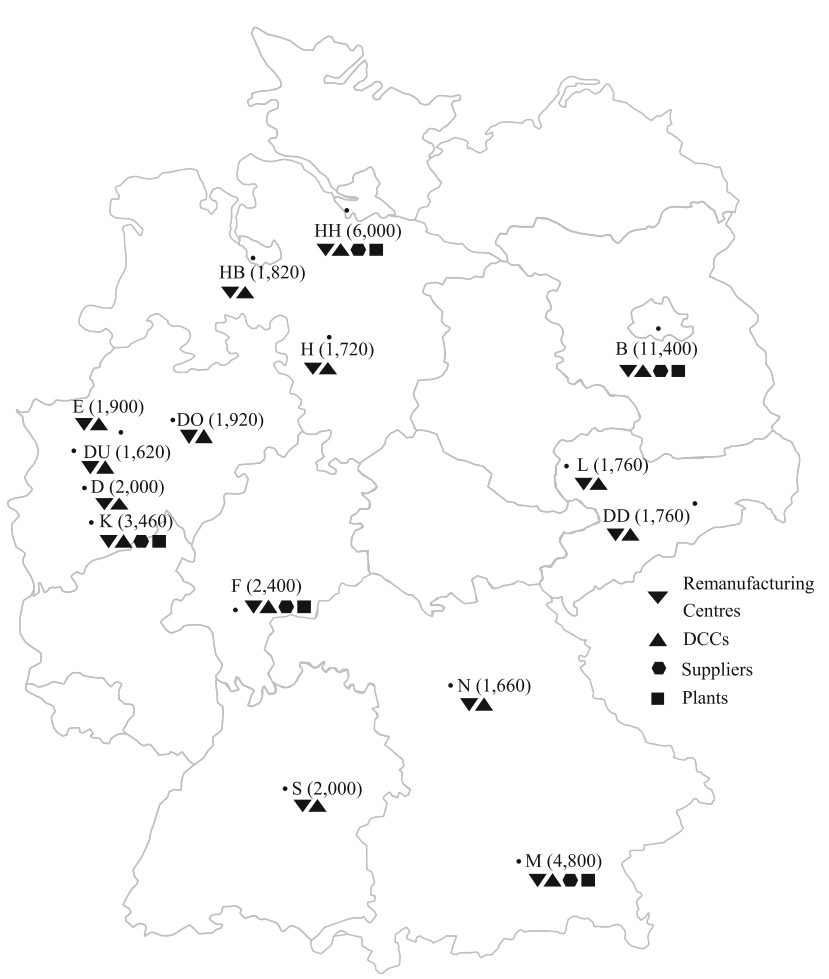

Fig. 2 Possible facility locations and the location of suppliers and customers with their respective demand 
The return rate $q_{k p s}^{t}$, where $(s, t) \in T: s \leq t$, is independent of customer location and product type, as in [8]. The multi-period modelling framework of the FLCAPPR model allows to model a temporal shift between the forward and reverse product flows in the network of $t-s=$ $m r$ periods, i.e. a residence time of a product with a customer can be defined. In this work a residence time of one year is assumed. Moreover, following [8] the return fraction is assumed to be $50 \%$, i.e. in the initial setting in this work, $50 \%$ of products shipped to the customers are returned at the beginning of the following period. Therefore, for 5 planning periods the return rate is

$$
\begin{aligned}
& q_{k p 1}^{1}=q_{k p 1}^{3}=q_{k p 1}^{4}=q_{k p 1}^{5}=q_{k p 2}^{2}=q_{k p 2}^{4}=q_{k p 2}^{5}=q_{k p 3}^{3} \\
& =q_{k p 3}^{5}=q_{k p 4}^{4}=q_{k p 5}^{5}=0 \\
& \text { and } q_{k p 1}^{2}=q_{k p 2}^{3}=q_{k p 3}^{4}=q_{k p 4}^{5}=0.5 .
\end{aligned}
$$

The other relevant parameters of the initial example and their values can be found in Table 4. The fraction of the returned products that has to be disposed, i.e. leaving the network, is 0.6, as stated in [8]. However, in the CLSCN studied in this work, returned products and components can be disposed; therefore, here the sum of the fractions of returned products $m d$ and components $m d^{c}$ that has to be disposed is set to 0.6, i.e. $m d=m d^{c}=0.3$. The transportation costs in the network $c_{v k}^{p}, c_{k v}^{p}, c_{v r}^{p}, c_{r f}^{c}, c_{z f}^{c}$ and $c_{f v}^{p}$ are taken from [8]. It is assumed that the costs for shipping goods between facilities of the same type are identical; the distances between the locations are taken from [10].

The cost difference between the procurement costs $c_{z}^{c}$ and remanufacturing costs $c_{r}$ is assumed as $10 \mathrm{MU}$ per component, as described in [8]. Disposal costs $c^{D E n t}$ and $s h_{y}^{x}, \forall y \in F \cup R, x \in C \cup P$ are taken from [8] as well.

Following Silver's [27] recommendations the inventory holding costs $h_{r}^{p}=h_{r}^{c}=h_{f}^{c}, \forall p \in P, r \in R, c \in C, f \in F$ are assumed as $0.25 \mathrm{MU}$ per unit per year.

The opening costs for the facilities, $f_{v}, f_{f}$ and $f_{r}$, are taken from [8]. The costs and revenues for capacity adjustments are based on these costs and assumed as $f_{c a p}{ }_{y}^{\text {Cost }}=f_{c a p} p_{y}^{\mathrm{Rev}}=1000$ MU. This assumption is discussed in the following sensitivity analysis.

The volume capacity can be adjusted in steps of 1000 units. The lower volume capacity limits at facilities are $1000 \mathrm{~m}^{3}$; an open facility has to be equipped at least with one volume capacity step. The capacity upper bound is chosen such that one open DCC, remanufacturing centre and plant is sufficient for the total product or component flow, respectively.

It is assumed that capacity in labour hours can be increased or decreased, respectively, by multiples of 1610 h. This results from the following calculation: in Germany contractual labour hours per week often are $35 \mathrm{~h}$. The holiday entitlement is six weeks per calendar year. Therefore, the number of labour hours of one worker per year is $(52-6)$ ("working" weeks in a year) 35 (hours per week) $=1610$ (hours per year), neglecting public holidays and downtime due to sickness.

The FLCAPPR problem with the described data setting can be solved by the optimization software Gurobi 6.5.0 on a two $3.10 \mathrm{GHz}$ Intel Xeon Processor E5-2687W and 128 GB RAM computer in 197.71 seconds. The total discounted costs are 19,534,389.05 MU.

In the solution, only one DCC and one plant are used; both are located in Berlin and are open during the total planning horizon. The open facilities and their capacity equipments in the planning periods can be found in Fig. 3 .

In every planning period products are shipped from the open DCC in Berlin to the customers to meet their demand. The products at the DCC originate from the plant in Berlin, where these product quantities are assembled in every period using components that are delivered from the supplier in Berlin. In Fig. 4 the product and component flows in the network are depicted.

The workforce and volume capacity at the open plant is at the same level for every period, see Fig. 3, because in every planning period the same amount of products and components, respectively, are processed at the plant.

Since in the second period customers start to return products at the DCC in Berlin, the workforce and volume capacity at the DCC is increased from the first to the second period, see Fig. 3. The customer demand and the amount of products which are returned by customers remain the same in the following periods, therefore, the workforce and volume capacity at the DCC stay at the same level for the remaining periods of the planning horizon.

Every returned product is collected in the DCC in Berlin and then disposed. There is no open remanufacturing centre in the network and no component remanufacturing takes place in any period.

It is difficult to compare the results of the initial example with previous studies of FLPs with reverse product flows, e.g. in [8], since the planning problem is extended in this work. Hence, additional parameters had to be introduced and, therefore, the planning problems differ.

However, it is to be noticed that the solution of the single-period facility location model presented in [8] recommends to open remanufacturing locations and that recovered products should be used to meet demand, which is different from the solution of the initial example of the FLCAPPR problem in this work. Due to the fact that in the multi-period approach taken here costs for volume capacity 
Table 4 Definition of parameter data

\begin{tabular}{|c|c|}
\hline Parameter & Value \\
\hline$\alpha$ & 0.01 \\
\hline $\operatorname{Cap}_{y t}^{O}$ & $500,000 \mathrm{~m}^{3}, \forall y \in F \cup R, t \in T$ \\
\hline $\mathrm{Cap}_{v t}^{O}$ & $800,000 \mathrm{~m}^{3}, \forall v \in V, t \in T$ \\
\hline $\operatorname{Cap}_{y}^{U}$ & $1000 \mathrm{~m}^{3}, \forall y \in F \cup R \cup V, t \in T$ \\
\hline$c_{z}^{c}$ & 10 MU per component, $\forall c \in C, z \in Z$ \\
\hline$c^{D E n t}$ & $2.5 \mathrm{MU}$ per unit \\
\hline$c_{k}^{U}$ & 1000 MU per unit, $\forall k \in K$ \\
\hline$c_{y w}^{c}$ & $0.0030 \mathrm{MU}$ per km, $\forall c \in C, y \in Z \cup R, w \in F$ \\
\hline$c_{v k}^{p}$ & $0.01 \mathrm{MU}$ per km, $\forall p \in P, v \in V, k \in K$ \\
\hline$c_{k v}^{p}$ & 0.005 MU per km, $\forall p \in P, v \in V, k \in K$ \\
\hline$c_{v r}^{p}$ & 0.003 MU per km, $\forall p \in P, v \in V, r \in R$ \\
\hline$c_{f v}^{p}$ & 0.0045 MU per km, $\forall p \in P, v \in V, f \in F$ \\
\hline$c_{f}$ & $1 \mathrm{MU}$ per unit, $\forall f \in F$ \\
\hline$c_{r}$ & 0 MU per unit, $\forall r \in R$ \\
\hline$d V_{p}$ & $0.5 \mathrm{~h}, \forall p \in P$ \\
\hline$d R_{c}$ & $2 \mathrm{~h}, \forall c \in C$ \\
\hline$d F_{p}$ & $1 \mathrm{~h}, \forall p \in P$ \\
\hline$e$ & $1000 \mathrm{~m}^{3}$ \\
\hline$f_{c a p}^{\text {Cost }}$ & $1000 \mathrm{MU}, \forall y \in F \cup V \cup R$ \\
\hline$f c a p_{y}^{\mathrm{Rev}}$ & $1000 \mathrm{MU}, \forall y \in F \cup V \cup R$ \\
\hline$f_{r}$ & $500,000 \mathrm{MU}, \forall r \in R$ \\
\hline$f_{f}$ & $5,000,000 \mathrm{MU}, \forall f \in F$ \\
\hline$f_{v}$ & $1,500,000 \mathrm{MU}, \forall v \in V$ \\
\hline$f_{t}^{y}$ & $10,000 \mathrm{MU}, \forall y \in F \cup V \cup R, t \in T$ \\
\hline$g_{p}$ & $10 \mathrm{~m}^{3}, \forall p \in P$ \\
\hline$g_{c}$ & $2 \mathrm{~m}^{3}, \forall c \in C$ \\
\hline$h_{r}^{x}$ & 0.25 MU per unit, $\forall x \in C \cup P, r \in R$ \\
\hline$h_{f}^{c}$ & $0.25 \mathrm{MU}$ per unit, $\forall c \in C, f \in F$ \\
\hline$L a b C a p_{y t}^{O}$ & $1,000,000 \mathrm{~h}, \forall y \in F \cup V \cup R, t \in T$ \\
\hline$l a b c c_{y}$ & 15 MU per hour, $\forall y \in F \cup V \cup R$ \\
\hline le & $1610 \mathrm{~h}$ \\
\hline$m d$ & 0.3 \\
\hline$m d^{c}$ & $0.3, \forall c \in C$ \\
\hline$m r$ & 1 \\
\hline$s f_{y}$ & $50,000 \mathrm{MU}, \forall y \in F \cup V \cup R$ \\
\hline$s h_{y}^{x}$ & 2.5 MU per unit, $\forall y \in F \cup R, x \in C \cup P$ \\
\hline
\end{tabular}




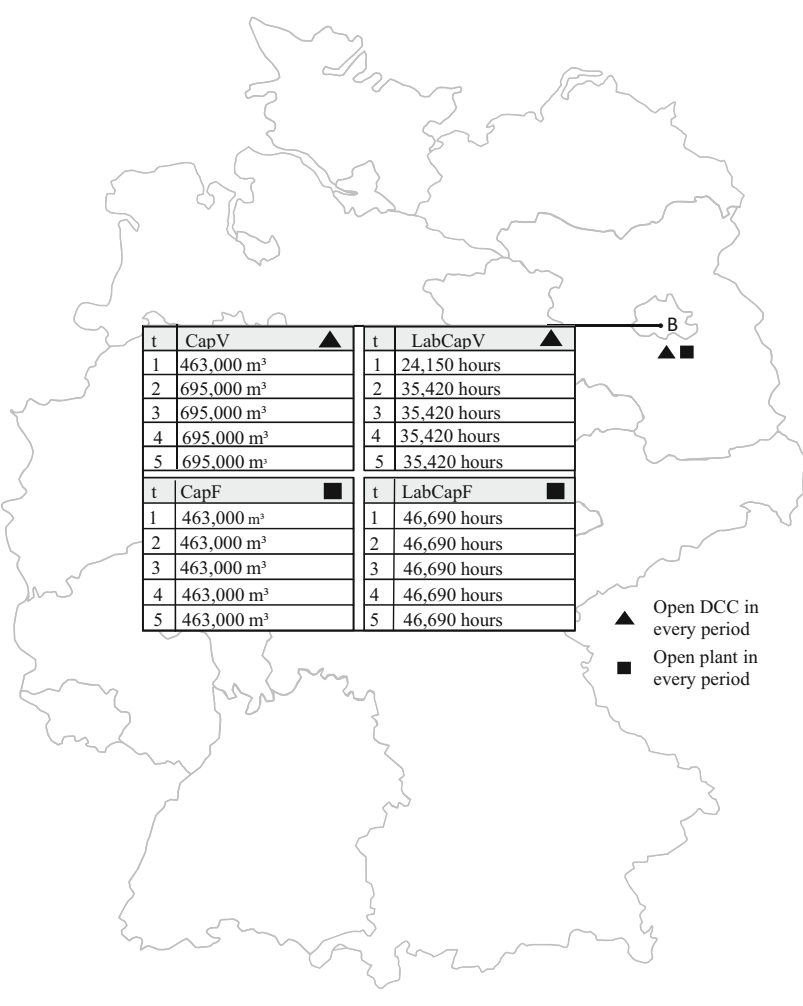

Fig. 3 Open facilities with their capacity levels

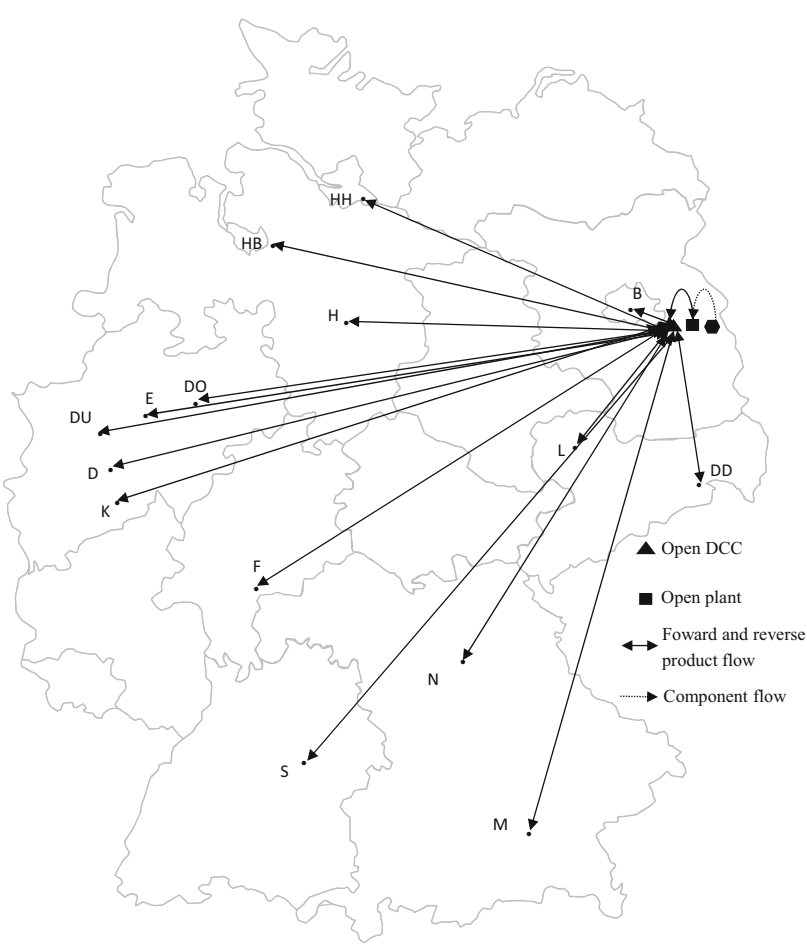

Fig. 4 Product and component flows in the network

and workforce are included which were not considered in [8] remanufacturing becomes less attractive and according to the FLCAPPR model, opening a remanufacturing centre and remanufacturing components is not cost-optimal.

\subsection{Sensitivity analysis}

In this section the sensitivity of the network structure to changes in the data is studied. First, the impact of the cost parameters on the decision to remanufacture is examined. Then the effect of the return rate on the network design is studied. Thereafter, the length of the planning horizon is varied and the influence on the network design and especially on the remanufacturing decision is discussed.

Due to rather extensive computing times the following tests were implemented allowing an optimality gap of up to $0.01 \%$. The exceptional cases are marked.

\subsubsection{Influence of cost parameters}

By extending the FLP to a multi-period planning problem in which facility locations, capacities and aggregate production are optimized, additional cost parameters are introduced in the objective function. In this section the impact of these cost parameters on the decision to remanufacture is studied.

Therefore, in the following fcap $_{r}^{\text {Cost }}$, labcc $_{r}, f_{t}^{r}$ and $c_{r}$, $\forall r \in R, t \in T$, i.e. the cost parameters for volume capacity and labour hours at the remanufacturing centres, for open remanufacturing centres and for remanufacturing, are varied, and the results are discussed.

Influence of volume capacity cost parameters

In previous facility location models in networks with product recovery, as i.e. in [8], the capacity level of a facility is assumed as given and it is not cost-optimally determined. Without taking into account costs for volume capacity, over-capacities in the network can occur.

Furthermore, the capacity requirements resulting from the flow of goods in a network with product recovery, especially the increased capacity requirements induced by the returned product flow additional to the requirements of forward product flows, are not considered as costs in the decision problem. In the FLCAPPR model building up volume capacity at a remanufacturing centre leads to costs; it is weighted with $f_{c a p}^{\text {Cost }}, \forall r \in R$, in the objective function.

In the initial example this cost is assumed as $1000 \mathrm{MU}$. Since in the solution of the initial example it is cost-optimal to dispose returned products and procure all components from a supplier, now the capacity cost is decreased to study if it has an impact on the remanufacturing decision.

The solution of the initial example stays optimal until $f_{c a p}{ }_{r}^{\text {Cost }}=400, \forall r \in R$. When $f_{c a p}{ }_{r}^{\text {Cost }} \leq 400$ MU, no reasonable solutions are obtained. Now, it is optimal to open every remanufacturing centre in periods $2-5$, and volume capacity is built up in one period and removed in the 
following period again and again over the planning horizon to gain the revenues from the volume capacity decrease, since $f c_{c a p}^{\mathrm{Rev}}=1000>400=f_{c}{ }^{\mathrm{R}}{ }_{r}^{\mathrm{Cost}}$. No returned products are processed at any remanufacturing centre. This solution is hardly realistic and shows that the parameters $f c a p_{r}^{\text {Cost }}$ and $f c a p_{r}^{\text {Rev }}$ have to be carefully determined.

Decreasing both parameters $f_{c a p}^{\text {Cost }}$ and $f c a p_{r}^{\text {Rev }}$ down to zero has no influence on the network, especially it remains optimal to dispose every returned product instead of opening remanufacturing centres and processing returned products.

\section{Influence of labour hour cost parameter}

In previous studies, as in [8], the workforce at facilities necessary for the respective operations at the facilities is not considered. However, the decisions to open a remanufacturing centre and to remanufacture components are interrelated. For remanufacturing, workforce at a remanufacturing centre is needed whose labour hours cost money. In this work, the workforce at facilities induces costs that are taken into account in the objective function.

It is assumed that a labour hour at the remanufacturing centre costs $l a b c c_{r}=15 \mathrm{MU}, \forall r \in R$. The impact of this cost parameter on the decision to remanufacture is studied in the following.

It turns out that for every value of $l a b c c_{r}>0$ the solution of the initial example remains cost-optimal. However, setting this parameter to zero has an impact on the network design. Now, it is cost-optimal to open a remanufacturing centre in the second period additional to the DCC and the plant in Berlin; the remanufacturing centre remains open for the remaining periods. The network and the open location with the respective capacity equipment are shown in Fig. 5.

The total discounted costs are 19,345,018.28 MU. With no labour hour cost at the remanufacturing centre, returned products are shipped to the remanufacturing centre. There returned products are stored or disassembled, and suitable components are remanufactured and used for product assembly at the plant. The processed and stored quantities are mapped in Figs. 6 and 7, respectively. In period 2-4 the distribution quantities between the DCC and remanufacturing centre are the same, in the last period the reverse product flow between the DCC and the remanufacturing centre is lower: less P2-products are transported to the remanufacturing centre. Since the quantity of products returned from customers stays the same from the second to the last period, the quantity of returned P2-products which are disposed is increased and at the remanufacturing centre less P2-products are disassembled in period 5. Therefore, over the total planning period just $24.48 \%$ of the components instead of the maximal possible $24.5 \%$ of the

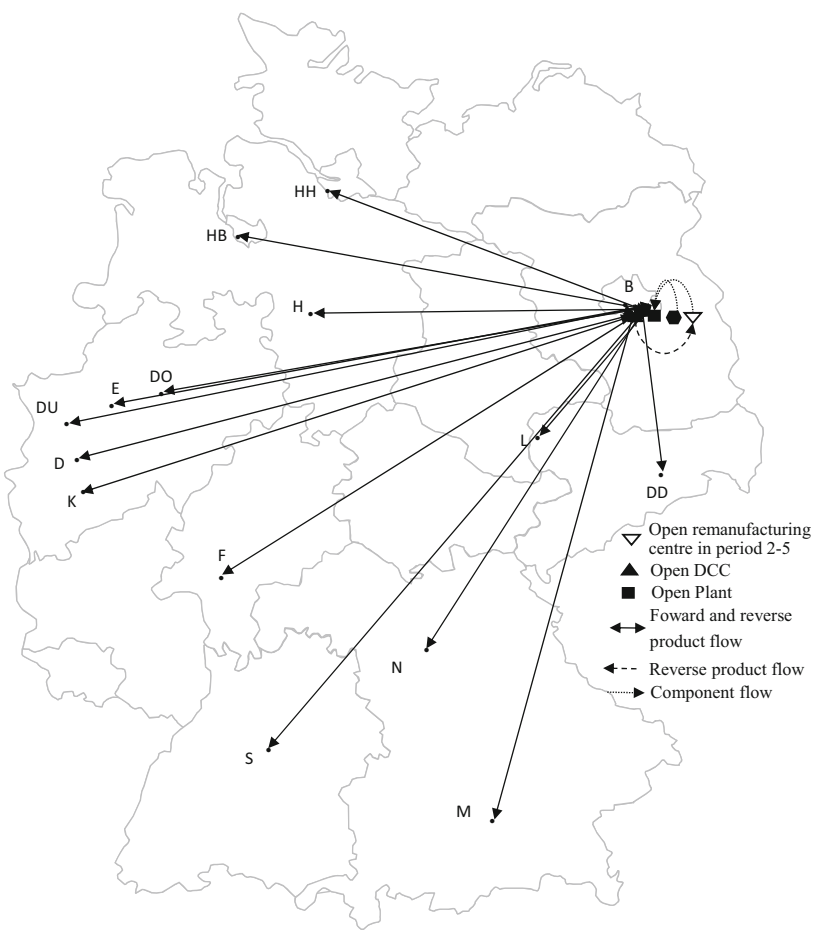

Fig. 5 Network with no labour hour costs for remanufacturing, $\operatorname{labcc}_{r}=0, \forall r \in R$

components used at the plant for product assembly are from the remanufacturing centre, the other components are procured from the supplier in Berlin. (The maximum percentage results from the limited number of returned products, which in turn results from the return rate.)

The workforce at the remanufacturing centre is set at the highest possible level in the second until the last period, see Fig. 8, because workforce induces no labour hour costs $\left(\right.$ labcc $\left._{r}=0\right)$.

The volume capacity at the remanufacturing centre is determined cost-optimally at $227,000 \mathrm{~m}^{3}$ for the second until the fourth period. In the last planning period the capacity is reduced by one step, thus $1000 \mathrm{~m}^{3}$, to obtain revenues for capacity reduction. To comply with this reduced volume capacity the returned product quantity shipped to the remanufacturing centre is slightly decreased, as the processed quantities at the remanufacturing centre, see Fig. 6. Hence, the decisions regarding the volume capacity level and the processed quantities at the remanufacturing centre and the reverse product flows in the network are interrelated.

The capacity equipments of the plant and the DCC in Berlin stay at the same levels as in the initial example, because the product flows between the plant and the DCC and between the DCC and the customers are not influenced by the labour hour costs at the remanufacturing centre. Furthermore, if a remanufacturing centre is opened, this 


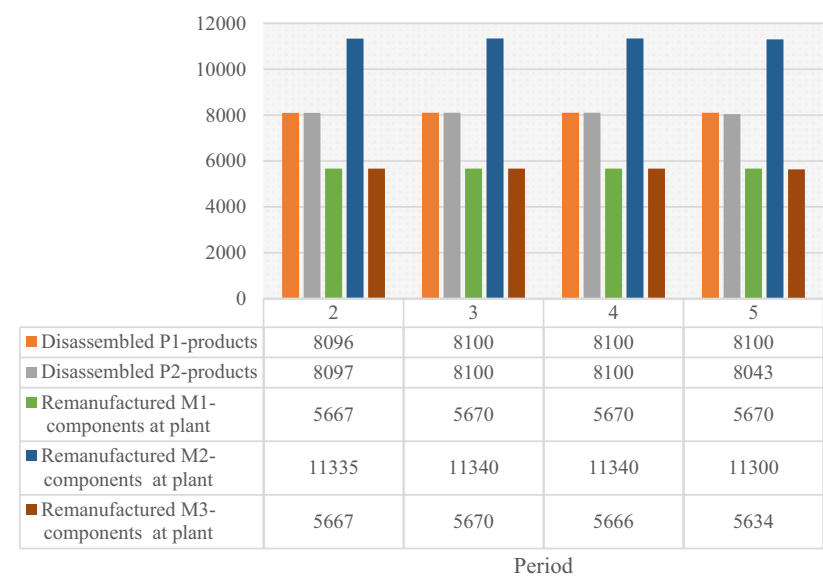

Fig. 6 Processed quantities at remanufacturing centre, labcc $_{r}=0, \forall r \in R$

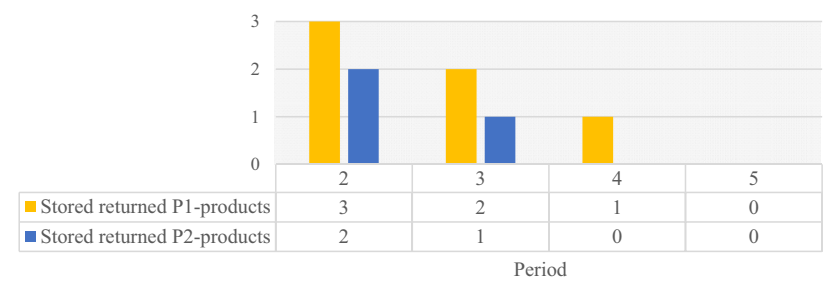

Fig. 7 Stored product quantities at remanufacturing centre, $l_{a b c c_{r}}=0, \forall r \in R$

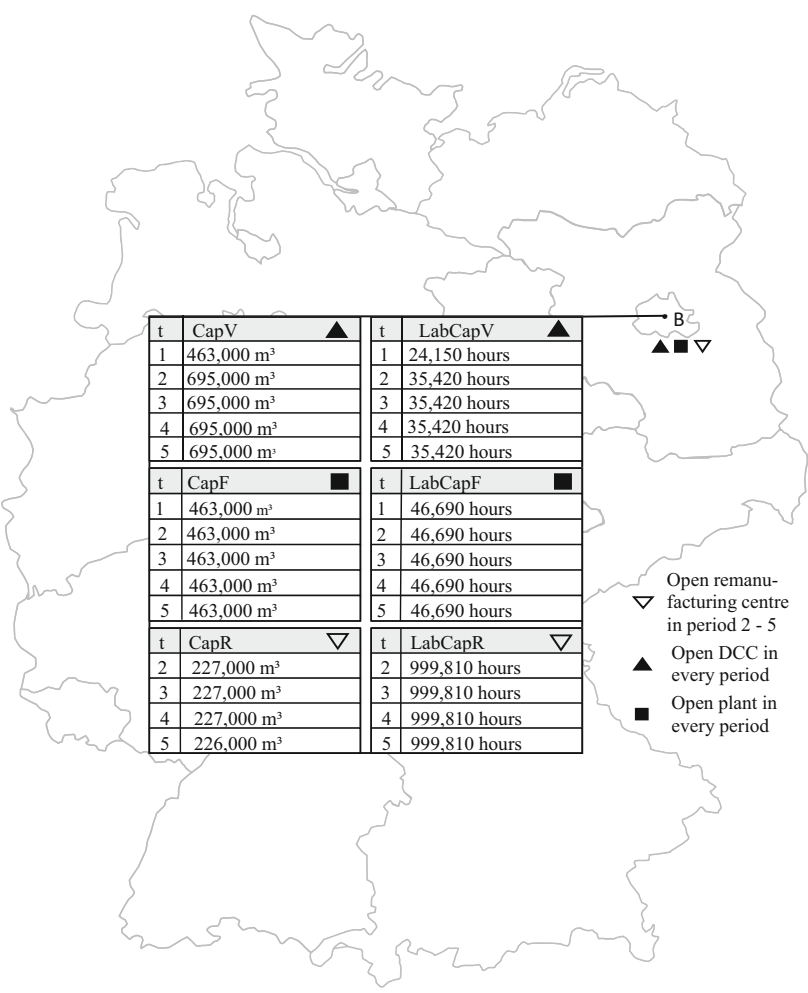

Fig. 8 Open facilities with their capacity levels for $l a b c c_{r}=0, \forall r \in R$ does not have an impact on the DCC and plant location nor on their capacity equipment.

\section{Influence of costs for open remanufacturing centres}

The FLCAPPR model optimizes the CLSCN over multiple periods. It is assumed that a facility in this network can be opened in any period and in the later periods the open facility can be closed or stay open.

The opening costs are taken from [8] and the cost per period for an open remanufacturing centre, $f_{t}^{r}, \forall r \in R, t \in T$, are assumed in the initial example as $10,000 \mathrm{MU}$. In the initial example no remanufacturing centre is opened. To study if and how this cost influences this decision, the cost is decreased until $f_{t}^{r}=0$.

At $f_{t}^{r}=0$ it is still not cost-optimal to open a remanufacturing centre. Hence, decreasing the cost parameter $f_{t}^{r}, \forall r \in R, t \in T$ has no impact on the decision to open a remanufacturing centre under this data setting.

\section{Influence of remanufacturing cost parameter}

As in [8], the difference between the remanufacturing and procurement costs $c_{r}, \forall r \in R$ and $c_{z}^{c}, \forall c \in C, z \in Z$, respectively, is assumed as $10 \mathrm{MU}$, that is $c_{r}=0, \forall r \in R$ and $c_{z}^{c}=10 \mathrm{MU}, \forall c \in C, z \in Z$. The solutions presented in [8] recommend remanufacturing of copiers unlike the solution of the initial example in this work. In this section it is studied, if and how the remanufacturing costs in relation to the procurement costs influence the remanufacturing decision.

The FLCAPPR problem is solved with different values for $c_{r}, \forall r \in R$. Since no remanufacturing takes place at $c_{r}=0, \forall r \in R$, just negative values of $c_{r}, \forall r \in R$ are studied, i.e. remanufacturing a component is subsidized.

For every $0>c_{r}>-30 \mathrm{MU}, \forall r \in R$ the solution remains the same as described in the solution of the initial example. From $c_{r}=-30 \mathrm{MU}, \forall r \in R$ it is cost-optimal to remanufacture. Hence, a difference of $40 \mathrm{MU}$ between the remanufacturing and procurement costs is necessary in order to make remanufactured components preferable to procured components. Because costs for volume capacity and workforce are included in the FLCAPPR model, this difference has to be bigger than in other studies where these costs are ignored, e.g. in [8]. At $c_{r}=-30 \mathrm{MU}, \forall r \in$ $R$ a remanufacturing centre in Berlin is opened in the second period and stays open in the remaining periods. Like in the solution of the initial example a DCC and a plant in Berlin are open in every planning period. The network and capacity levels at open facilities are mapped in Fig. 9. The total discounted costs are 19,346,946.96 MU, i.e. costs can be slightly decreased.

The capacity level and product flows between the plant and the DCC and the DCC and the customers remain as in 


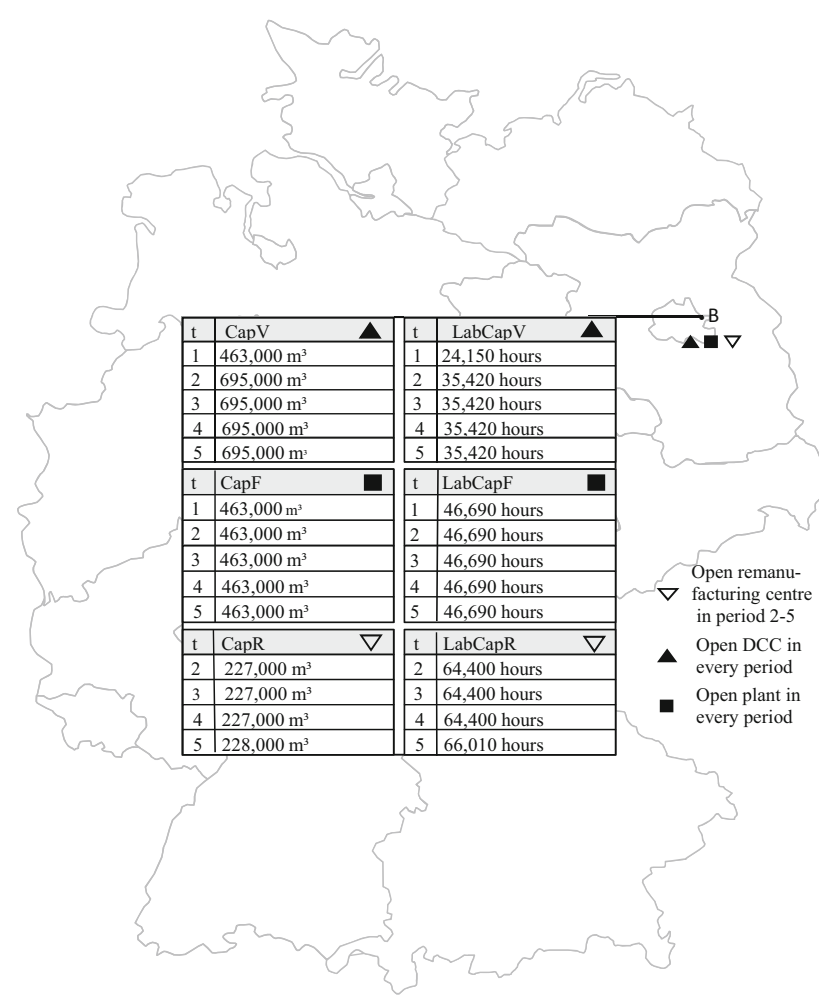

Fig. 9 Network with negative remanufacturing costs, $c_{r}=-30, \forall r \in R$

the solution of the initial example, see Fig. 4. However, there is a product flow between the DCC and the remanufacturing centre in Berlin, as shown in Fig. 5.

From the second until the last period products collected at the DCC are shipped to the remanufacturing centre. At the remanufacturing centre the returned products are stored and disassembled to components and suitable components are remanufactured. After remanufacturing, the components are shipped to the plant. The processed and stored quantities at the remanufacturing centre are mapped in Figs. 10 and 11, respectively.

Every remanufacturable returned product and component is processed at the remanufacturing centre and then shipped from the remanufacturing centre to the plant, i.e. there are no items on inventories at the end of period 5.

In addition to remanufactured components, components have to be procured from the supplier in Berlin; only $24.5 \%$ of the components used in the product assembly are remanufactured components. Compared to the solution of the previous section for $l a b c c_{r}=0, \forall r \in R$, this fraction is slightly increased. For $c_{r}=-30, \forall r \in R$ every remanufacturable product is processed at the remanufacturing centre.

There is an interrelation between the decisions to store and process products and the capacity equipment at the remanufacturing centre, as the capacity levels at the

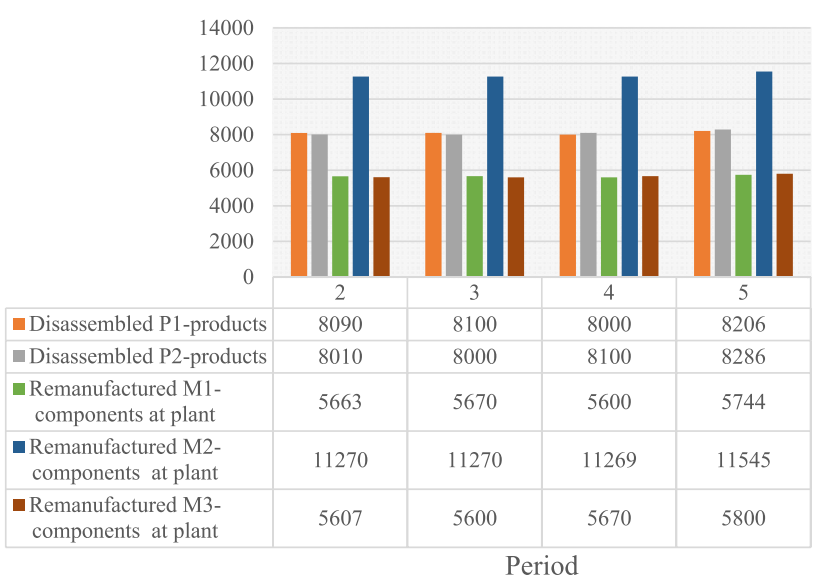

Fig. 10 Processed quantities at remanufacturing centre, $c_{r}=-30, \forall r \in R$

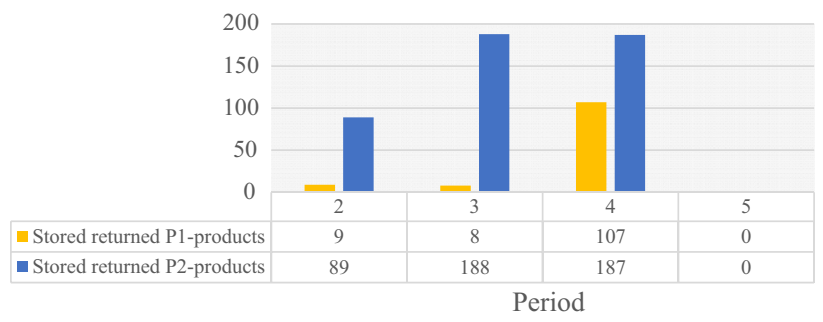

Fig. 11 Stored product quantities at remanufacturing centre, $c_{r}=-30, \forall r \in R$

remanufacturing centre are adapted to the requirements of the processed and stored quantities, see Figs. 10 and 11. By storing products from the second to the fourth period instead of processing them immediately at the remanufacturing centre, less workforce is necessary at the remanufacturing centre in these periods, see Fig. 9. However, due to building up inventory at the remanufacturing centre from the second until the fourth period, more volume capacity is necessary in the fifth period; in the last period the capacity has to be increased to comply with the increased volume requirements. In the last planning period all items on inventory are processed, therefore, the workforce in the last period is increased, too, see Fig. 9.

\subsubsection{Influence of return rate}

The FLCAPPR model is solved with different values for $q_{k p s}^{t}, \forall k \in K, p \in P,(s, t) \in T: s \leq t$, i.e. with return rates from 0 to 1 , increasing in steps of 0.1 . The respective total discounted costs and selected decision variable values for the results can be found in Fig. 12.

When there are no returned products at all, a plant and DCC are opened in Berlin, but of course no remanufacturing centre is opened. The total discounted costs are 


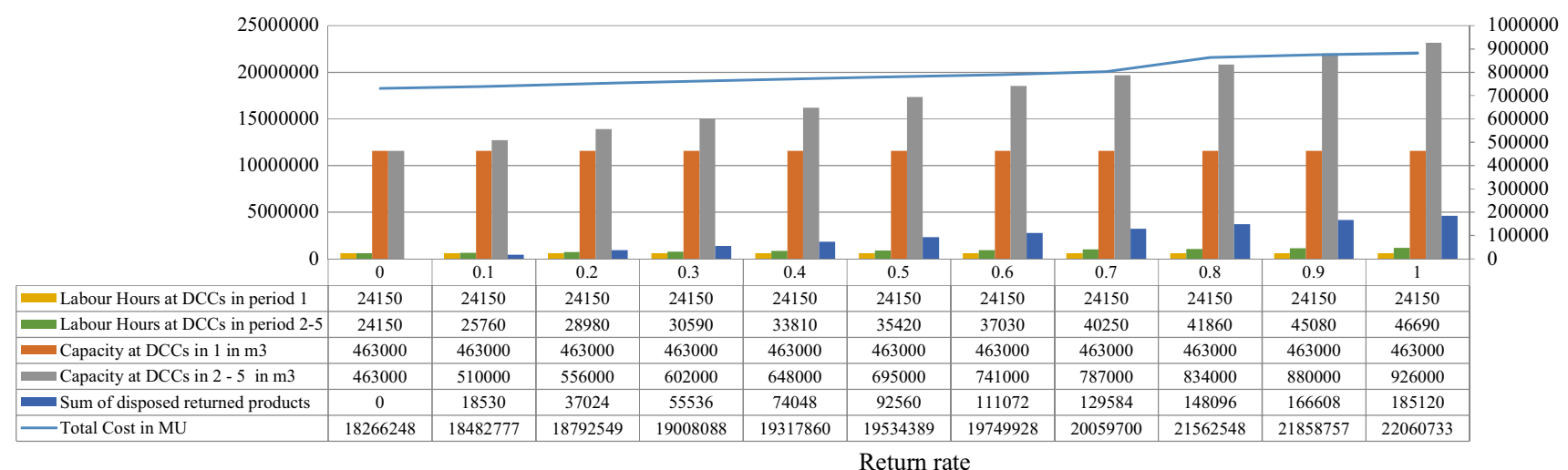

Fig. 12 Variation of return rate with $c_{r}=0, \forall r \in R$

18, 266, 247.79 MU and, hence, lower than before, as no transportation and other costs for returned products occur.

For every return rate from 0 to 1 , the decision regarding the opening and the capacity equipment of the plant remains the same. Moreover, for every return rate no remanufacturing centre is opened, every returned product is disposed and every component used in the product assembly is procured from the supplier in Berlin.

The total discounted costs of the CLSCN increase with the return rate, see Fig. 12. This cost increase is induced by higher capacity and by disposal and opening costs due to an increased quantity of returned products which are collected in the DCCs and disposed afterwards.

Due to the assumption that returned products have to be collected at DCCs, the return rate has an influence on the capacity equipment at the DCC and the opening decision regarding DCCs, as every returned product flows through a DCC and requires handling times and space, see Fig. 12. For a return rate between 0.8 and 1 the volume capacity of one DCC is not sufficient any more; therefore, a DCC in Dortmund is opened additional to the DCC in Berlin. The resulting network with the product and component flows is displayed in Fig. 13.

From the DCC in Dortmund products are shipped to customers in Dortmund, Duisberg, Düsseldorf, Essen, Frankfurt am Main and Cologne in every planning period, and in the first period the demand of customers in Stuttgart is met by products from the DCC in Dortmund. The remaining customers receive their products from the DCC in Berlin.

Some reverse product flows are different from the forward product flows. Customers in Bremen and Hannover return their products to the DCC in Dortmund, although they get products from the Berlin DCC. The other customers return their products to the DCC which delivered the product.

The results show that the reverse product flow influences the forward product flow and the optimal network

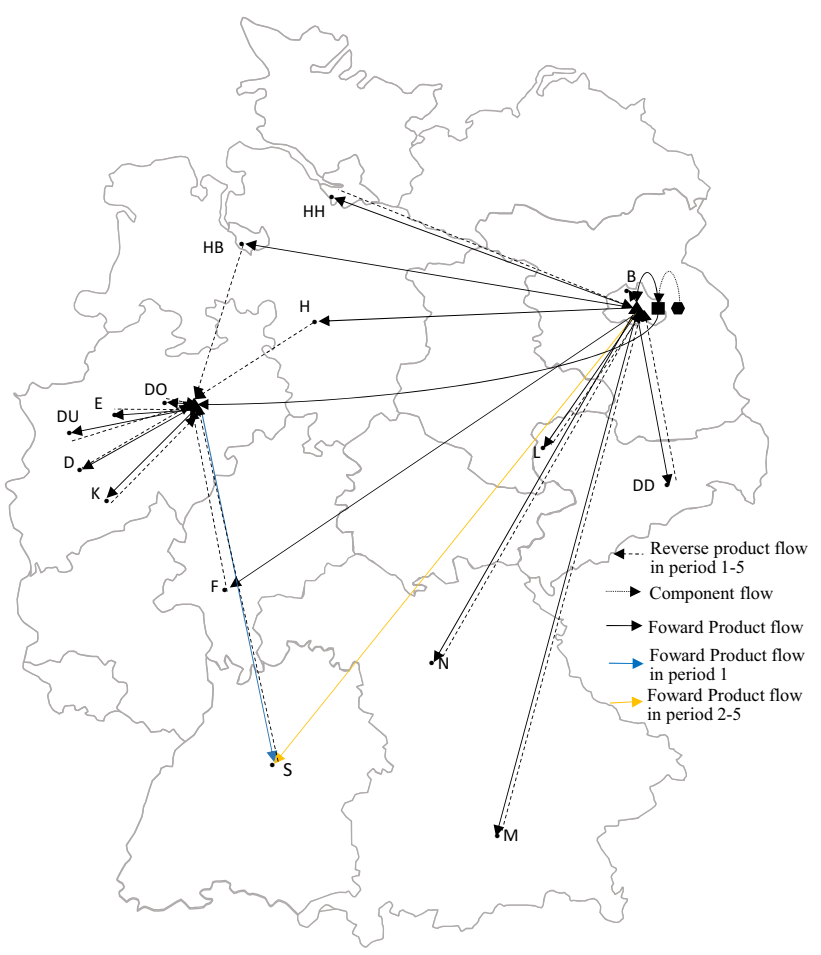

Fig. 13 Network with two DCCs for $q_{k p s}^{t}=\{0.8,0.9,1\}$

structure, if the quantity of returned products is high, as in [8]. Therefore, the forward and reverse product flows have to be planned simultaneously in order to achieve an optimal network.

Moreover, it turns out that a higher quantity of returned products increases the total costs, but does not necessarily result in a network with remanufacturing. There is no return rate at which it is cost-optimal to open a remanufacturing centre and use remanufactured components in the product assembly under the initial data setting, in particular for the assumed cost parameters.

Now, it is examined which results occur when it is costoptimal to open a remanufacturing centre and to remanufacture all suitable components and the return rate is 


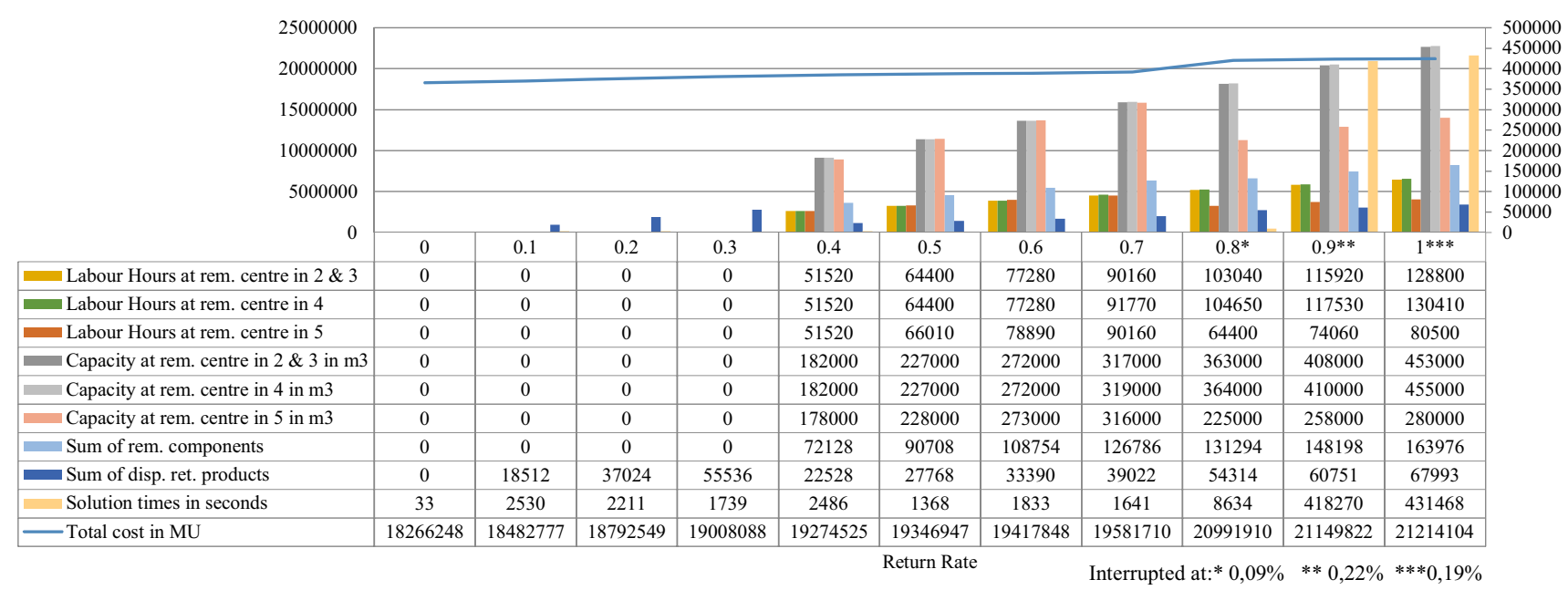

Fig. 14 Variation of return rate with $c_{r}=-30, \forall r \in R$

varied. Therefore, the FLCAPPR model is solved with $c_{r}=$ $-30, \forall r \in R$ and for return rates from 0 to 1 , increasing in steps of 0.1 . The respective total discounted costs of the resulting network and selected decision variable values for the results can be seen in Fig. 14.

The total discounted costs increase with the return rate, like in the study with $c_{r}=0, \forall r \in R$. However, for $c_{r}=$ $-30, \forall r \in R$ the cost increase for $0.4 \leq q_{k p s}^{t} \leq 1$ is slightly smaller, see Fig. 15.

At return rates $q_{k p s}^{t}<0.4$, every returned product is disposed and the network consists of a DCC and plant in Berlin, as in the solution of the initial example. Unlike in the study with $c_{r}=0$, with $c_{r}=-30$ an increase of the return rate has an impact on the decision to open a remanufacturing centre and to remanufacture components. For values of $q_{k p s}^{t}=0.4$ to $q_{k p s}^{t}=1$, a remanufacturing centre in Berlin is opened in the second period which stays open over the remaining planning periods. For these return rates, the respective maximal possible amount of components is remanufactured and used in the product assembly instead of procured components. Because remanufactured components are cheaper production inputs than procured

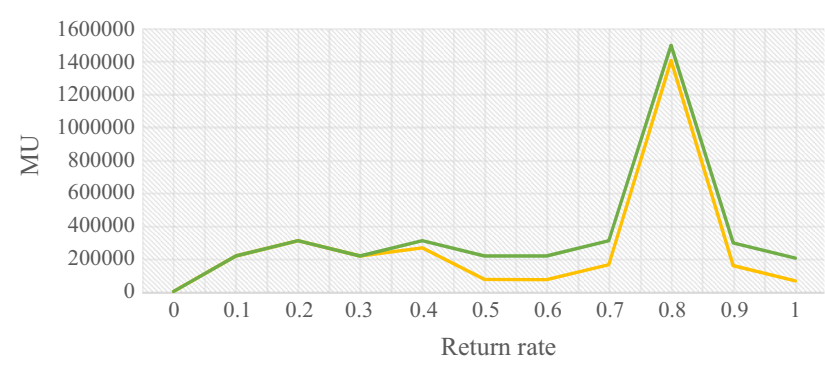

Cost increase with $\mathrm{cr}=-30 \quad$ Cost increase with $\mathrm{cr}=0$

Fig. 15 Cost increase by variation of return rate with $c_{r}=0, \forall r \in R$ and $c_{r}=-30, \forall r \in R$ components, for these return rates the total cost increase in the study with $c_{r}=-30$ is smaller than for the study with $c_{r}=0$ where no remanufacturing takes place, see Fig. 15 .

For $0.4 \leq q_{k p s}^{t} \leq 0.7$, it is cost-optimal to ship returned products from the DCC in Berlin to the remanufacturing centre in Berlin. The remanufactured components are used in the product assembly in the plant in Berlin. The network for these return rates was shown already in Fig. 5 .

As can be seen in Fig. 14, the capacity level at the remanufacturing centre increases with the return rate, i.e. with the quantity of returned products. Over the planning periods the capacity levels at the remanufacturing centre can change, too. This happens when returned products are stored and remanufactured in a later period, instead of being remanufactured immediately.

The capacity levels at the DCC increase with the return rate, too. At $q_{k p s}^{t}=\{0.8,0.9,1\}$ two DCCs, a DCC in Berlin and Dortmund, have to be opened to have enough capacity for the returned products, as in the study with $c_{r}=0$. The opening costs for the second DCC cause the jump in the total costs, see Fig. 15. The location of the remanufacturing centre and the plant stays at Berlin.

This analysis has shown that opening a remanufacturing centre and using remanufactured components in the product assembly is cost-optimal under specific remanufacturing costs and only if the return rate is high enough, $q_{k p s}^{t} \geq 0.4$. Moreover, high return rates, $q_{k p s}^{t}=\{0.8,0.9,1\}$, influence the network design and the distribution system, whether remanufacturing is cost-optimal or not.

\subsubsection{Influence of the number of planning periods}

In contrast to previous facility location models with reverse product flows the FLCAPPR model optimizes the CLSCN over multiple periods instead of a single period. A period is 
assumed as one year, and the length of the planning horizon can be interpreted as the product life-cycle. In the initial example the CLSCN is planned over 5 periods. In this section, the impact of the length of the planning horizon on the network is studied; therefore, the number of periods is varied.

The total discounted costs, the satisfied product demand and the disposed returned products over a planning horizon of 2-7 periods are listed in Fig. 16.

It is assumed that demand and return rate remain the same over the planning horizon. The structure of the solution of the initial example remains optimal, i.e. the network stays the same for an increased number of periods in the planning horizon. Especially, the decision to procure the components for the product assembly instead of remanufacturing components stays the same for the different length of the planning horizon under this data setting.

The capacity costs and the costs for open facilities rise when the number of periods is increased. Moreover, since products and components have to be transported, processed and procured, respectively, these costs increase with the number of planning periods, too. Hence, the total discounted costs grow when the planning periods are increased, see Fig. 16.

It is examined if the length of the planning horizon does not influence the network design and the production and stored quantities in the network, if remanufacturing is costoptimal. Therefore, the FLCAPPR model is solved with $c_{r}=-30, \forall r \in R$ for planning horizons of 2-7 periods. The total discounted costs and selected interesting decision variables are mapped in Fig. 17.

Increasing the planning horizon to six and seven periods results in the same network as in the solution with 5 periods, which was already described above. In this solution, remanufacturing takes place at an open remanufacturing centre in Berlin, see Fig. 5.

Decreasing the planning horizon of 5 periods by just one period, to 4 periods, changes the network design. Now, it is cost-optimal to procure every component for the product assembly from the supplier in Berlin instead of remanufacturing components. The resulting network is as in the solution of the initial example, where only a DCC and plant in Berlin are opened and both stay open over the planning horizon.

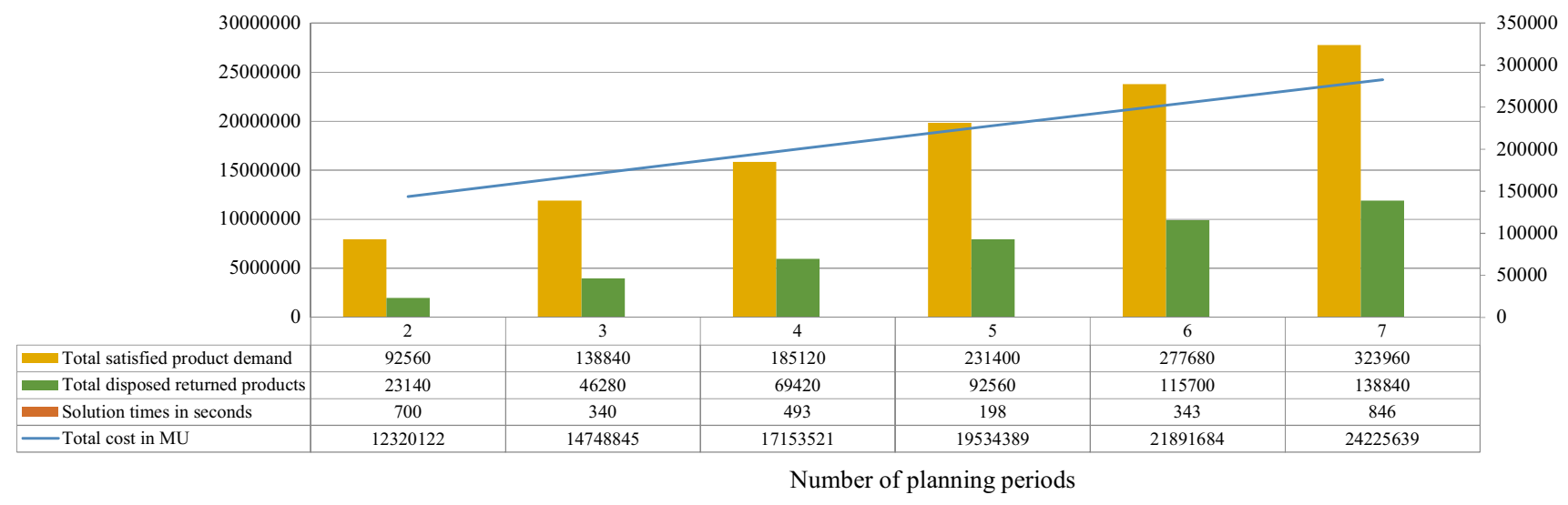

Fig. 16 Variation of planning horizon length with $c_{r}=0, \forall r \in R$

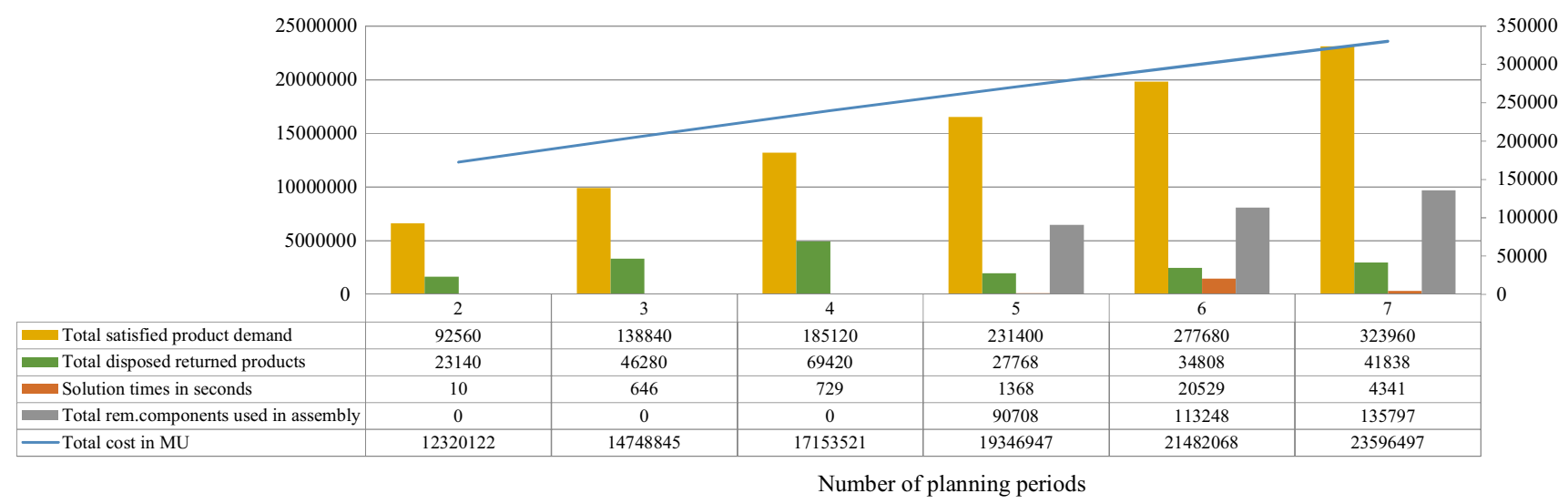

Fig. 17 Variation of planning horizon length with $c_{r}=-30, \forall r \in R$ 
Hence, the number of periods in the planning horizon has an impact on the network design and the decision to remanufacture. A multi-period planning approach in contrast to a single-period approach, as in [8], for a CLSCN is useful and leads to new and different results. Therefore, the relevant number of periods has to be determined and the network has to be analysed over this planning horizon.

\section{Conclusions and future research directions}

In this work, the strategic CLSCN design is extended by capacity and production planning on an aggregate level. The resulting FLCAPPR model determines the cost-optimal network design, the facility locations and capacity equipment at open facilities, and the cost-optimal procurement, production and distribution quantities in the CLSCN over a finite planning horizon consisting of multiple periods. It is solved for an example from the copier industry with input data based on previously published research [8]. Furthermore, possible effects of the extended planning approach on the network design, especially on the decision to recover returned products, are studied in a sensitivity analysis. Thereafter, the robustness of the network design and the production and distribution quantities regarding the return rate, i.e. the quantity of returned products, is examined. In a further study the influence of the planning horizon length is investigated.

Extending a single-period FLP to the FLCAPPR model leads to new and different results concerning the decision to remanufacture and the effect of the return rate on the network. In contrast to a previous study [8], in this setting, it is cost-optimal to dispose returned products instead of recovering them and use the resulting components in the product assembly based on the FLCAPPR model. When capacity costs, especially labour hour costs at the remanufacturing centre, are included, remanufacturing is costoptimal only if the remanufacturing costs are sufficiently low compared to the procurement costs for new components from suppliers. Hence, production costs, in particular remanufacturing costs, have a large impact on facility location and capacity equipment decisions. Furthermore, the interdependence between the capacity equipment at the remanufacturing centre and the procuring decision, the processing and storing quantities at the remanufacturing centre is determined. Hence, these decisions have to be optimized jointly, as in the FLCAPPR model.

The decisions regarding facility locations and capacity equipment are robust for low return rates. From return rates of $40 \%$ remanufacturing takes place, if remanufacturing costs are sufficiently low. Moreover, for high return rates, rates of $80 \%$ and more, the facility location decisions and the distribution system are changed compared to the results for lower rates. Therefore, the forward and reverse product flows in the network have to be planned together.

The multi-period setting of the FLCAPPR model enables a study of the influence of the planning horizon on the decision to remanufacture and provides new information compared to single-period FLPs, as in [8]. Product recovery is cost-optimal only if remanufacturing costs are sufficiently low compared to procurement costs and the planning horizon is sufficiently long. For the studied data setting, this means that there has to be product demand for at least 5 periods, i.e. five years; only then remanufacturing can be cost-optimal.

Following the CLSC-Management definition in [12], a CLSCN has to be studied over the total product life-cycle with consideration of varying demand and returned product quantities and qualities. Hence, in the future the FLCAPPR problem should be solved with data sets consisting of varying demand and returned product quantities in order to study possible product life-cycle effects.

Moreover, when residence times are longer than one period, the reverse product flow starts later in the planning horizon. This can affect decisions regarding the facility locations and capacity equipment and, therefore, the remanufacturing decision. Hence, different assumptions regarding the residence times of products should be examined in the future.

Linear costs and revenues for adjusting capacity are assumed here, and the effects of economies of scale and learning effects gained by higher production quantities and bigger facilities are not considered. Furthermore, the possibilities to increase or decrease labour hours at facilities by overtime or part-time, respectively, is not modelled. These aspects require further model extensions, but it has to be noted that the FLCAPPR model is already large scale with rather lengthy solving times.

Finally, uncertainties regarding the quantity and quality of reverse product flows are an issue of CLSCM [11-13]. They are not considered here, because an APP framework is used. However, other approaches integrating uncertainties might be developed; this is left for future research.

Open Access This article is distributed under the terms of the Creative Commons Attribution 4.0 International License (http://crea tivecommons.org/licenses/by/4.0/), which permits unrestricted use, distribution, and reproduction in any medium, provided you give appropriate credit to the original author(s) and the source, provide a link to the Creative Commons license, and indicate if changes were made.

\section{References}

1. Akcali E, Cetinkaya S, Uster H (2009) Network design for reverse and closed-loop supply chains: an annotated bibliography of models and solution approaches. Networks 53:231-248 
2. Akinc U, Khumawala BM (1977) An efficient branch and bound algorithm for the capacitated warehouse location problem. Manag Sci 23:585-594

3. Akinc U, Roodman GMA (1986) New approach to aggregate production planning. IIE Trans 18:88-94

4. Bostel N, Dejax P, Lu Z (2005) The design, planning, and optimization of reverse logistics networks, logistics systems: design and optimization. Springer, New York, pp 171-212

5. Fleischmann B, Meyr H, Wagner M (2015) Advanced planning. Supply chain management and advanced planning. Springer, Berlin

6. Fleischmann M, Bloemhof-Ruwaard JM, Dekker R, van der Laan E, van Nunen JAEE, Van Wassenhove LN (1997) Quantitative models for reverse logistics: a review. Eur J Oper Res 103:1-17

7. Fleischmann M, Krikke HR, Dekker R, Flapper SDP (2000) A characterisation of logistics networks for product recovery. Omega 28:653-666

8. Fleischmann M, Beullens P, Bloemhof-Ruwaard JM, Van Wassenhove LN (2001) The impact of product recovery on logistics network design. Prod Oper Manag 10:156-173

9. Fleischmann M, Bloemhof-Ruwaard JM, Beullens P, Dekker R (2004) Reverse logistics network design. Reverse logistics: quantitative models for closed-loop supply chains. Springer, Berlin

10. Google Maps: Google Maps. https://maps.google.com. Version: 2015, checked on: 12.01.2015

11. Guide VDR Jr (2000) Production planning and control for remanufacturing: industry practice and research needs. J Oper Manag 18:467-483

12. Guide VDR Jr (2009) The evolution of closed-loop supply chain research. Oper Res 57:10-18

13. Guide VDR, Jayaraman V, Srivastava R (1999) Production planning and control for remanufacturing: a state-of-the-art survey. Robot Comput Integr Manuf 15:221-230

14. Jayaraman V (1998) Transportation. Facility location and inventory issues in distribution network design. Int J Oper Prod Manag 18:471-494

15. Jayaraman V (1999) A closed-loop logistics model for remanufacturing. J Oper Res Soc 50:497-508

16. Jayaraman V (2006) Production planning for closed-loop supply chains with product recovery and reuse: an analytical approach. Int J Prod Res 44:981-998

17. King A, Miemczyk J, Bufton D (2006) Photocopier remanufacturing at Xerox UK. A description of the process and consideration of future policy issues. Innovation in life cycle engineering and sustainable development. Springer, Dordrecht

18. Lund RT (1998) Remanufacturing: an American resource. International Congress Environmentally Conscious Design and Manufacturing, Rochester

19. Marín A, Pelegrín B (1998) The return plant location problem: modelling and resolution. Eur J Oper Res 104:375-392

20. Nam S, Logendran R (1992) Aggregate production planning-a survey of models and methodologies. Eur J Oper Res 61:255-272

21. Perl J, Sirisoponsilp S (1988) Distribution networks: facility location. Transportation and inventory. Int J Phys Distrib Mater Manag 18:18-26

22. Pishvaee MS, Torabi SA (2010) A possibilistic programming approach for closed-loop supply chain network design under uncertainty. Fuzzy Sets Syst 161:2668-2683

23. Salema MIG, Barbosa-Povoa AP, Novais AQ (2007) An optimization model for the design of a capacitated multi-product reverse logistics network with uncertainty. Eur J Oper Res 179:1063-1077

24. Schultmann F, Engels B, Rentz O (2003) Closed-loop supply chains for spent batteries. Interfaces 33:57-71

25. Schultmann F, Zumkeller M, Rentz O (2006) Modeling reverse logistic tasks within closed-loop supply chains: an example from the automotive industry. Eur J Oper Res 171:1033-1050

26. Shen ZM (2007) Integrated supply chain design models: a survey and future research directions. J Ind Manag Optim 3:1-27

27. Silver EA (1967) A tutorial on production smoothing and work force balancing. Oper Res 15:985-1010

28. Souza GC (2013) Closed-loop supply chains: a critical review, and future research. Dec Sci 44:7-38

29. Statistische Ämter des Bundes und der Länder: Gemeindeverzeichnis-Sonderveröffentlichung Gebietsstand: 31.12.2011 (Jahr): Großstädte (mit mindestens 100000 Einwohnerinnen und Einwohnern) in Deutschland nach Bevölkerung am 31.12.2011 auf Grundlage des Zensus 2011 und früherer Zählungen. https:// www.destatis.de/DE/ZahlenFakten/ LaenderRegionen/Regionales/Gemeindeverzeichnis/ Administrativ/GrosstaedteEinwohner.html. Accessed on 01/15/2015

30. Steinke L, Fischer K (2015) Integrated facility location, capacity, and production planning in a multi-commodity closed supply chain network. Logistic management. Springer, Berlin 